\title{
Topological obstructions to fatness
}

\author{
LUIS A FLORIT \\ WOLFGANG ZILLER
}

\begin{abstract}
Alan Weinstein showed that certain characteristic numbers of any Riemannian submersion with totally geodesic fibers and positive vertizontal curvatures are nonzero. In this paper we explicitly compute these invariants in terms of Chern and Pontrjagin numbers of the bundle. This allows us to show that many bundles do not admit such metrics.
\end{abstract}

\section{C20, 57R20, 57R22}

There are few known examples of compact Riemannian manifolds with positive sectional curvature; see Ziller [22] for a survey. All of them, apart from some rank one symmetric spaces, can be viewed as the total space of a Riemannian submersion, in some cases an orbifold submersion; see Florit and Ziller [7]. The fact that the homogeneous ones also have totally geodesic fibers motivated A Weinstein [19] to study Riemannian submersions with totally geodesic fibers and positive vertizontal curvatures, ie, sectional curvatures of planes spanned by a vector tangent to a fiber and a vector orthogonal to it. He called such fiber bundles fat, and showed that this much weaker condition already imposes strong restrictions.

Fat circle bundles are in one-to-one correspondence with symplectic manifolds and hence well understood. Therefore, we will restrict ourselves to bundles whose fiber dimension is bigger than one, which in turn implies that the dimension of the base must be divisible by 4 .

Let $G \rightarrow P \stackrel{\pi}{\rightarrow} B^{2 m}$ be a $G$-principal bundle with $G$ a compact connected Lie group endowed with a biinvariant metric $\langle$,$\rangle , and B$ a compact connected manifold. Given a principal connection $\theta$ with curvature form $\Omega$, we say that $\theta$ is $u$-fat if

$$
\Omega_{u}=\langle\Omega(\cdot, \cdot), u\rangle \text { is nondegenerate on } \mathcal{H},
$$

where $\mathcal{H}$ is the horizontal space of $\pi$ and $u$ lies in the Lie algebra $\mathfrak{g}$ of $G$. For a connection metric on $P$ (see Section 1) this condition is equivalent to requiring that the sectional curvatures spanned by the action field generated by $u$ and any horizontal vector is positive. In particular, fatness is independent of the metrics on the base and fiber. 
If fatness holds for all $0 \neq u \in \mathfrak{g}$ we say that $\theta$ is fat, or simply the principal bundle is fat. If it holds for all $u \neq 0$ in a subset $\mathfrak{s} \subset \mathfrak{g}$, we say that the principal connection is $\mathfrak{s}-f a t$, or that $\mathfrak{s}$ is fat. Observe that if $u$ is fat, so are all vectors in its adjoint orbit $\mathfrak{o}=\operatorname{Ad}_{G}(u)$. Following Weinstein, we consider the homogeneous $\operatorname{Ad}_{G}$-invariant polynomial $q_{\mathfrak{o}}: \mathfrak{g} \rightarrow \mathbb{R}$ defined as

$$
q_{\mathfrak{o}}(\alpha)=\int_{G}\left\langle\operatorname{Ad}_{g}(u), \alpha\right\rangle^{m} d g .
$$

By Chern-Weil theory, there exists a closed $2 m$-form $\omega_{\mathfrak{o}}$ on $B^{2 m}$ such that $\pi^{*} \omega_{\mathfrak{o}}=$ $q_{\mathfrak{o}}(\Omega)$. By $\mathfrak{o}$-fatness, the form $\left\langle\operatorname{Ad}_{g}(u), \Omega\right\rangle^{m}$ is everywhere nonzero on $\mathcal{H}$ and thus $\omega_{\mathfrak{o}}$ is a volume form on $B^{2 m}$. Therefore, the characteristic number $\int_{B} \omega_{\mathfrak{o}}$ is nonzero and we call it the Weinstein invariant associated to $\mathfrak{o}$.

Our main purpose is to compute these invariants for the classical Lie groups, obtaining explicit topological obstructions to fatness in terms of Chern (or Pontrjagin) numbers. This will allow us to derive several applications.

The simplest case is the torus $T^{n}$ for which we obtain a lower bound on the Betti numbers of the base.

Theorem 1 Let $T^{n} \rightarrow P \rightarrow B^{2 m}$ be a fat principal bundle. Then, the Betti numbers of $B^{2 m}$ satisfy $b_{2 i} \geq n$ for all $1 \leq i \leq m-1$.

Denote by $c_{k} \in H^{2 k}(B, \mathbb{Z})$ and $p_{k} \in H^{4 k}(B, \mathbb{Z})$ the Chern and Pontrjagin classes, and by $e \in H^{2 n}(B, \mathbb{Z})$ the Euler class when $G=\mathrm{SO}(2 n)$. In the case of $\mathrm{U}(2)$ and $\mathrm{SO}(4)$, Weinstein invariants have rather simple expressions.

Theorem 2 Let $G \rightarrow P \rightarrow B^{2 m}$ be a fat principal bundle.

(a) If $G=\mathrm{U}(2)$, then $c_{1}^{m}$ and $\left(c_{1}^{2}-4 c_{2}\right)^{m / 2}$ are nonzero and have the same sign, and

$$
\sum_{j=0}^{m / 2}\left(\begin{array}{c}
m+1 \\
2 j+1
\end{array}\right) t^{2 j} c_{1}^{m-2 j}\left(c_{1}^{2}-4 c_{2}\right)^{j} \neq 0 \quad \forall t \in \mathbb{R} .
$$

(b) If $G=\mathrm{SO}(4)$, then $\left(p_{1}+2 e\right)^{m / 2}$ and $\left(p_{1}-2 e\right)^{m / 2}$ are nonzero and have the same sign, and

$$
\sum_{j=0}^{m / 2}\left(\begin{array}{c}
m+2 \\
2 j+1
\end{array}\right) t^{2 j}\left(p_{1}-2 e\right)^{m / 2-j}\left(p_{1}+2 e\right)^{j} \neq 0 \quad \forall t \in \mathbb{R} .
$$


For Lie groups with $\operatorname{rank}(G)>2$ the formulas become more complicated, eg in the case of $\mathrm{U}(n)$ we obtain

$\sum_{n \geq \lambda_{1} \geq \cdots \geq \lambda_{m} \geq 0} \prod_{i=1}^{m}\left(n+m-i-\lambda_{i}\right) ! \operatorname{det}\left(\sigma_{\lambda_{i}+j-i}(y)\right)_{1 \leq i, j \leq m} \operatorname{det}\left(c_{\lambda_{i}+j-i}\right)_{1 \leq i, j \leq m} \neq 0$ for all $0 \neq y=\left(y_{1}, \ldots, y_{n}\right)$, with $\sum \lambda_{i}=m$, where $\sigma_{i}$ stands for the elementary symmetric polynomial of degree $i$ in $n$ variables. Observe that special consequences are $c_{1}^{m} \neq 0$ for $y=(1, \ldots, 1)$ and $\operatorname{det}\left(c_{j-i+1}\right)_{1 \leq i, j \leq m} \neq 0$ for $y=(1,0, \ldots, 0)$. Similar formulas hold for the other classical Lie groups; see Theorem 2.3.

We use these invariants as follows. Given a $G$-principal bundle $P$, the Weinstein invariants define homogeneous polynomials in $\operatorname{rank}(G)$ variables once we parametrize the adjoint orbits $\mathfrak{o} \subset \mathfrak{g}$ in terms of a maximal abelian subalgebra $\mathfrak{t} \subset \mathfrak{g}$, by writing $\mathfrak{o}=\operatorname{Ad}_{G}(y)$ for $y \in \mathfrak{t}$. The coefficients of these multivariable polynomials are Chern numbers of $P$, and fatness implies that they have no nonzero real roots. It is thus in general difficult to express the nonvanishing of the Weinstein invariants in terms of the Chern numbers alone. But in some cases this is possible. For example, we have the following which applies, in particular, to base manifolds with $b_{4}\left(B^{2 m}\right)=1$.

Corollary 1 Let $G \rightarrow P \rightarrow B^{2 m}$ be a principal bundle, where $G=\mathrm{U}(2)$ or $G=$ $\mathrm{SO}(4)$. Suppose there exists $r \in \mathbb{R}$ such that $c_{1}^{2}-4 c_{2}=r c_{1}^{2} \neq 0$ if $G=\mathrm{U}(2)$, or $p_{1}+2 e=r\left(p_{1}-2 e\right) \neq 0$ for $G=\mathrm{SO}(4)$. Then all Weinstein invariants are nonzero if and only if $r>0$.

A natural context where partial fatness appears is for associated bundles. Given $H \subset G$ a closed subgroup, we have the associated bundle by $G / H \rightarrow P \times{ }_{G} G / H=P / H \rightarrow B$. A connection metric on this fiber bundle can be described in terms of a principal connection on $P$. The vertizontal curvatures of such a connection metric are positive if and only if the principal connection on $P$ is $\mathfrak{h}^{\perp}$-fat. It turns out that any fat bundle is associated to some principal bundle in this way.

Perhaps the most natural examples of associated bundles are the sphere bundles. In the real case, we conclude from Theorem 2.3 the following.

Corollary 2 A sphere bundle with totally geodesic fibers and positive vertizontal curvatures satisfies $\operatorname{det}\left(p_{j-i+1}\right)_{1 \leq i, j \leq m / 2} \neq 0$, where $2 m$ is the dimension of the base.

We will see that if the sphere bundle is the sphere bundle of a complex or quaternionic vector bundle, we obtain a one parameter family of obstructions instead of a single one. 
Derdziński and Rigas [5] showed that the only $\mathbb{S}^{3}$ bundle over $\mathbb{S}^{4}$ which admits a fat connection metric is the Hopf bundle. For $\mathbb{S}^{7}$ bundles over $\mathbb{S}^{8}$ this is still an open problem. Such bundles $\mathbb{S}^{7} \rightarrow M_{k, l} \rightarrow \mathbb{S}^{8}$ are classified by two arbitrary integers $k, l$ such that $p_{2}=6(k-l)$ and $e=k+l$. Using the obstructions for quaternionic sphere bundles and Corollary 2, we will show:

Corollary 3 The sphere bundles $\mathbb{S}^{7} \rightarrow M_{k, l} \rightarrow \mathbb{S}^{8}$, where either $k=l$ or $(k, l)=$ $(8 r, 4 r), r \in \mathbb{Z}$, do not admit a fat connection metric. In particular, for $k=l=1$, it follows that $T_{1} \mathbb{S}^{8} \rightarrow \mathbb{S}^{8}$ does not admit a fat connection metric.

Similarly, we will see that $T_{1} \mathbb{C} P^{4} \rightarrow \mathbb{C} P^{4}$ admits no fat connection metric. For fat $\mathbb{S}^{3}$-fiber bundles over $\mathbb{C} P^{2}$ we have the following.

Corollary 4 The only two $\mathbb{S}^{3}$-fiber bundles over $\mathbb{C} P^{2}$ that may admit a fat connection metric are the complex sphere bundles with $c_{1}^{2}=9$ and $c_{2}=1$ or 2 . In particular, $T_{1} \mathbb{C} P^{2} \rightarrow \mathbb{C} P^{2}$ does not have a fat connection metric.

Bérard-Bergery classified in [1] the fat fiber bundles which are homogeneous. A family of such examples are the fiber bundles over the Grassmannian of 2-planes in $\mathbb{C}^{n+1}$,

$$
\mathrm{U}(2) / S_{p, q}^{1} \rightarrow \mathrm{U}(n+1) / \mathrm{U}(n-1) \cdot S_{p, q}^{1} \rightarrow G_{2}\left(\mathbb{C}^{n+1}\right),
$$

where $S_{p, q}^{1}=\left\{\operatorname{diag}\left(z^{p}, z^{q}\right) \in \mathrm{U}(2): z \in S^{1}\right\}$. The fiber is the lens space $\mathbb{S}^{3} / \mathbb{Z}_{p+q}$ when $p+q \neq 0$. He showed that the bundle has a homogeneous fat connection metric if and only if $p q>0$. We will show that the homogeneity property can be dropped:

Corollary 5 The above fiber bundles with $p q \leq 0$ admit no fat connection metric.

For general $\mathrm{U}(2) / S_{p, q}^{1}$ fiber bundles we have:

Corollary 6 If $\mathrm{U}(2) / S_{p, q}^{1} \rightarrow P \rightarrow B^{2 m}$ is a fat bundle, then $\left(c_{1}^{2}-4 c_{2}\right)^{m / 2} \neq 0$. Moreover, if $(p, q) \neq(1,1)$ and $c_{1}^{2}=r\left(c_{1}^{2}-4 c_{2}\right)$ for some $r \in \mathbb{R}$, then the nonvanishing of the Weinstein invariants is equivalent to

$$
r>-\left(\frac{1-\cos \left(\frac{\pi}{m+1}\right)}{1+\cos \left(\frac{\pi}{m+1}\right)}\right)\left(\frac{p+q}{p-q}\right)^{2} .
$$

The above fat bundles over $G_{2}\left(\mathbb{C}^{n+1}\right)$ show that Corollary 6 is sharp, since for $n=2$ these bundles are associated to the same principal bundle $\mathrm{U}(2) \rightarrow \mathrm{SU}(3) \rightarrow \mathbb{C} P^{2}$ which has $r=-1 / 3$. 
In Section 1 we collect various facts about fat fiber bundles and the algebra of symmetric polynomials and Schur functions. These turn out to be central in converting the integrals into polynomials in Chern and Pontrjagin numbers. In Section 2 we derive the general form of Weinstein invariants for all classical Lie groups and for $G_{2}$. In Section 3 we concentrate on the case of $G=T^{n}, \mathrm{U}(2)$ and $\mathrm{SO}(4)$, while in Section 4 we discuss sphere bundles. In Section 5 we prove a stronger version of the reduction conjecture stated by the second author [21] for normal subgroups, namely, no fat vector exists in $\mathfrak{h}^{\perp}$ if the structure group reduces to a normal subgroup with Lie algebra $\mathfrak{h}$. Finally, in Section 6 we relate our obstructions to some of the fat bundles in [1] by computing their Weinstein invariants. Throughout the paper we will provide several additional applications.

Acknowledgements We would like to thank N Wallach for helpful conversations.

Both authors were partially supported by CNPq-Brazil and the second one by a grant from the National Science Foundation.

\section{Preliminaries}

We first recall Weinstein's definition of fatness of a fiber bundle and his basic topological obstruction to fatness; see Weinstein [19] and Ziller [21].

Let $\pi: M \rightarrow B$ be a fiber bundle with fiber $F$, and metrics on $M$ and $B$ such that $\pi$ is a Riemannian submersion. Let $\mathcal{H}$ and $\mathcal{V}$ denote the horizontal and vertical subbundles of $T M$. If the fibers of $\pi$ are totally geodesic, the sectional curvature of a vertizontal 2-plane, ie a plane spanned by a vertical vector $U$ and horizontal vector $X$ is equal to $\left\|A_{U} X\right\|^{2}$, where $A: \mathcal{H} \times \mathcal{H} \rightarrow \mathcal{V}$ is the O'Neill tensor and $\left\langle A_{U} Y, X\right\rangle=-\left\langle A_{X} Y, U\right\rangle$. In particular, these curvatures are automatically nonnegative. According to Weinstein,

$\pi: M \rightarrow B$ is called fat if $A_{X} U \neq 0$ for all $0 \neq X \in \mathcal{H}, 0 \neq U \in \mathcal{V}$,

or, equivalently, when all vertizontal sectional curvatures are positive.

We first consider the case where the fiber bundle $\tau: P \rightarrow B$ is a $G$-principal bundle and the horizontal spaces are $G$-invariant. The horizontal distribution can then be described in terms of a principal connection $\theta: T P \rightarrow \mathfrak{g}$ as $\mathcal{H}=\operatorname{ker} \theta$, where $\mathfrak{g}$ is the Lie algebra of $G$. With the aid of a metric on the base and a left invariant metric on $G, \theta$ defines a so called connection metric on $P$ by declaring $\mathcal{H}$ and $\mathcal{V}$ to be orthogonal, endowing $\mathcal{H}$ with the pull back of the metric on the base, and $\mathcal{V}$ with the chosen left invariant metric on $G$. If we endow $P$ with such a connection metric and 
$\Omega: T P \times T P \rightarrow \mathfrak{g}$ is the curvature form of $\theta$, Weinstein observed that fatness of $\tau$ can be rewritten as:

(1.1) For each $0 \neq y \in \mathfrak{g}, \Omega_{y}:=\langle\Omega(\cdot, \cdot), y\rangle$ is a nondegenerate 2-form on $\mathcal{H}$,

where we have chosen an auxiliary biinvariant metric $\langle\cdot, \cdot\rangle$ on $G$. This is indeed an immediate consequence of $2 \theta\left(A_{X} Y\right)=-\Omega(X, Y)$. In particular (1.1) implies that fatness is independent of the metrics on the base and fiber, ie, it only depends on the principal connection itself. We thus simply say that the principal connection $\theta$, or $P$ by abuse of language, is fat. Furthermore, if $\mathfrak{s} \subseteq \mathfrak{g}$ is a subset, we will say that $\theta$ is $\mathfrak{s}-f a t$ if $\Omega_{y}$ is nondegenerate for all $0 \neq y \in \mathfrak{s}$. Also observe that if a vector $y \in \mathfrak{g}$ is fat, the whole adjoint orbit $\operatorname{Ad}_{G}(y)$ consists of fat vectors since $\Omega_{\operatorname{Ad}_{g}(y)}=g^{*}\left(\Omega_{y}\right)$. Hence we can assume that $y$ lies in a maximal abelian subalgebra $\mathfrak{t} \subseteq \mathfrak{g}$.

Observe that for each fat vector $y \in \mathfrak{g}$ we have a nonvanishing vector field $Z_{y}$ on the unit sphere of $\mathcal{H}$ given by $\left\langle Z_{y}(X), Y\right\rangle=\Omega_{y}(X, Y)$, and if $\left\{y_{1}, \ldots, y_{r}\right\}$ is a basis of a fat subspace $V \subset \mathfrak{g}$, the vector fields $Z_{y_{1}}, \ldots, Z_{y_{r}}$ are pointwise linearly independent. By the well known Radon-Hurwitz formula $V$-fatness thus implies:

$$
\text { If } \operatorname{dim} B=(2 a+1) 2^{4 b+c} \text { with } 0 \leq c \leq 3 \text {, then } \operatorname{dim} V \leq 2^{c}+8 b-1 .
$$

In particular,

$$
\text { If } \operatorname{dim} V \geq 2,4,8 \text {, then } 4|\operatorname{dim} B, 8| \operatorname{dim} B, 16 \mid \operatorname{dim} B \text {, respectively. }
$$

Notice that the adjoint orbit of $V$ may contain a linear subspace of larger dimension, in some cases all of $\mathfrak{g}$, which gives further restrictions.

We define the Weinstein invariants as follows, where we assume that $G$ and $B$ are compact and connected. For each adjoint orbit $\mathfrak{o} \subseteq \mathfrak{g}$, we write $\mathfrak{o}=\operatorname{Ad}_{G}(y)$ for $y \in \mathfrak{t}$. For $k \in \mathbb{N} \cup\{0\}$, the homogeneous $\operatorname{Ad}_{G}$-invariant polynomial $q_{y}^{k}=q_{\mathfrak{o}}^{k}: \mathfrak{g} \rightarrow \mathbb{R}$ given by

$$
q_{\mathfrak{o}}^{k}(\alpha)=\int_{G}\left\langle\operatorname{Ad}_{g}(y), \alpha\right\rangle^{k} d g
$$

defines a closed $2 k$-form $\omega_{\mathfrak{o}}$ on $B^{2 m}$ via $\tau^{*} \omega_{\mathfrak{o}}=q_{\mathfrak{o}}^{k}(\Omega)$. By Chern-Weyl theory, $\left[w_{\mathfrak{o}}\right] \in H^{2 k}(B, \mathbb{R})$ represents a characteristic class of the bundle. Now suppose that $k=m$ is half the dimension of the base and write $q_{\mathfrak{o}}=q_{\mathfrak{o}}^{m}$. If $\mathfrak{o}$ is fat, $\Omega_{y}^{m} \neq 0$ is a volume form on $\mathcal{H}$. Thus, if $G$ is connected, $\left\langle\operatorname{Ad}_{g}(y), \Omega\right\rangle^{m}$ is nowhere zero and has constant sign when $g$ varies along $G$, and the integral $q_{\mathfrak{o}}(\Omega)$ is nonzero on $\mathcal{H}$. Hence $\omega_{\mathfrak{o}}$ is a volume form of $B^{2 m}$, in particular $B^{2 m}$ is orientable, and the characteristic number $\int_{B} \omega_{\mathfrak{o}}$ is nonzero. We call this characteristic number the Weinstein invariant associated to $\mathfrak{o}$, and our main goal is to express it explicitly in terms of Chern and Pontrjagin numbers. This will allow us to obtain various applications. 
Observe that, for a circle bundle, fatness is equivalent to $\omega$ being a symplectic form on the base, where $\omega$ is given by $\Omega=\tau^{*} w$. Thus, the only Weinstein invariant is the symplectic volume. For any other fat fiber bundle, by (1.3) we have that 4 divides the dimension of the base. Therefore, we always assume that $m$ is even.

For convenience, we use the same notation for an $\operatorname{Ad}_{G}$-invariant polynomial on $\mathfrak{g}$, for its restriction to a maximal abelian subalgebra $\mathfrak{t} \subseteq \mathfrak{g}$, and for the corresponding characteristic class. Recall that the Chern classes $c_{i} \in H^{2 i}(B, \mathbb{Z})$ are defined by the $\operatorname{Ad}_{\mathrm{U}(n)}$-invariant polynomials $c_{i}(A)$, with

$$
\operatorname{det}(I+t A)=\sum_{i} c_{i}(A) t^{i}, \quad A \in \mathfrak{u}(n)
$$

and the Pontrjagin classes $p_{i} \in H^{4 i}(B, \mathbb{Z})$ by the $\operatorname{Ad}_{\mathrm{O}(n)}$-invariant polynomials $p_{i}(A)$, with

$$
\operatorname{det}(I+t A)=\sum_{i} p_{i}(A) t^{2 i}, \quad A \in \mathfrak{o}(n)
$$

For even rank, we also have the Euler class $e \in H^{2 n}(B, \mathbb{Z})$ given by the $\operatorname{Ad}_{\mathrm{SO}(2 n)^{-}}$ invariant Pfaffian

$$
e(A)=\operatorname{Pf}(A), \quad A \in \mathfrak{o}(2 n),
$$

where $\operatorname{Pf}^{2}(A)=\operatorname{det}(A)$. Again, by abuse of notation, we also use the same symbol for the quaternionic Pontrjagin classes $p_{i} \in H^{4 i}(B, \mathbb{Z})$ given by the $\operatorname{Ad}_{\mathbb{S}(n) \text {-invariant }}$ polynomials $p_{i}(A)$ as in (1.5), but for $A \in \mathfrak{s p}(n)$. As it is well known, these polynomials form a basis of the set of all $\operatorname{Ad}_{G}$-invariant polynomials in the case of a classical Lie group $G$, the only relations being $e^{2}=p_{n}$ in the case of $G=\operatorname{SO}(2 n)$ and $c_{1}=0$ for $G=\mathrm{SU}(n)$. Thus each Weinstein invariant is a polynomial in these basic classes, $c_{i}$, $p_{i}$ and $e$, evaluated on the fundamental cycle $[B]$.

Now, as a function of $\mathfrak{o}=\operatorname{Ad}_{G}(y), q_{\mathfrak{o}}=q_{y}$ becomes a polynomial in $y \in \mathfrak{t} \cong \mathbb{R}^{n}$, with coefficients being Chern or Pontrjagin numbers. By definition they are invariant under the Weyl group $W=N(T) / T$. We use the following standard forms for $\mathfrak{t}$ : for $\mathfrak{u}(n)$ and $\mathfrak{s p}(n)$ we have $\mathfrak{t}=\left\{i \operatorname{diag}\left(y_{1}, \ldots, y_{n}\right): y \in \mathbb{R}^{n}\right\}$, while for $\mathfrak{s o}(2 n+1)$ and $\mathfrak{s o}(2 n)$ we have $\mathfrak{t}=\left\{\operatorname{diag}\left(y_{1} J, \ldots, y_{n} J\right): y \in \mathbb{R}^{n}\right\}$, where $J$ stands for the basic $2 \times 2$ skew symmetric matrix. We will denote both the vector and its coordinates by $y=\left(y_{1}, \ldots, y_{n}\right) \in \mathfrak{t}$. Since all Weyl groups of the classical Lie groups contain the permutation group, $q_{y}$ can be expressed in terms of the elementary symmetric polynomials $\sigma_{i}(y)=\sigma_{i}\left(y_{1}, \ldots, y_{n}\right)$. We will choose the biinvariant metric on $G$ in such a way that the canonical basis in $\mathbb{R}^{n} \cong \mathfrak{t}$ is orthonormal. 
To obtain the invariants it was important for $G$ to be a connected compact Lie group. We claim that the obstructions also hold for nonconnected groups. To see this we lift the bundle to a certain cover of the base whose structure group reduces to a connected Lie group. Let $\Gamma=G / G_{o}$ be the component group of the Lie group, on which $G$ acts naturally. Let $\bar{B}$ be a connected component of $\{(b, \gamma) \mid b \in B, \gamma \in \Gamma\}$ and define the cover $\alpha: \bar{B} \rightarrow B$ by $\alpha((b, \gamma))=b$. This induces the pull back bundle $\alpha^{*}(P)=$ $\{(x, b, \gamma) \in P \times \bar{B} \mid \sigma(x)=b\}$ on which $G$ acts via $g(x, b, \gamma)=\left(x g^{-1}, b, g \gamma\right)$. There now exists a reduction $\bar{P}=\{(x, b, e)\} \subset \alpha^{*}(P)$ which is preserved by $G_{o}$. The connection $\theta$ on $P$ pulls back to a connection $\bar{\theta}$ on $\bar{P}$ and its curvature $\bar{\Omega}$ still satisfies the property that $\bar{\Omega}_{y}$ is a nondegenerate 2 -form on $\mathcal{H}$ for all $0 \neq y \in \mathfrak{g}$. Thus, if $\bar{q}_{\mathfrak{o}}(\alpha)=\int_{G_{o}}\left\langle\operatorname{Ad}_{g}(y), \alpha\right\rangle^{m} d g$, we have $\bar{q}_{\mathfrak{o}}(\bar{\Omega})=\bar{\tau}^{*}\left(\bar{\omega}_{\mathfrak{o}}\right)$ with $\bar{\omega}_{\mathfrak{o}}$ a volume form on $\bar{B}$, and $\bar{\omega}_{\mathfrak{o}}=\alpha^{*}\left(\omega_{\mathfrak{o}}\right)$. Therefore, $\omega_{\mathfrak{o}}$ is also a volume form and $\int_{B} \omega_{\mathfrak{o}} \neq 0$.

We now study how Weinstein invariants behave for coverings. Let $\widetilde{G}$ be a finite cover of a connected Lie group $G, G=\widetilde{G} / \Gamma$, and assume that $P$ has a cover $\varphi: \widetilde{P} \rightarrow P$ which is a $\widetilde{G}$-principal bundle. Due to the fact that $\Gamma$ is a subgroup of the center of $G,(1.4)$ is invariant under $\Gamma$ and therefore the Weinstein invariants for $\widetilde{P}$ are precisely the ones for $P$ multiplied by the order of $\Gamma$. Moreover, observe that a principal connection $\theta$ on $P$ is fat if and only if $\varphi^{*} \theta$ on $\widetilde{P}$ is fat.

Similarly, if $G \rightarrow G / \Gamma=G^{*}$ is a covering, a $G$ principal bundle $P \rightarrow B$ induces a $G^{*}$ principal bundle $P / \Gamma \rightarrow B$. If $\theta$ is a fat connection on $P$, there exists a connection $\theta^{*}$ on $P / \Gamma$ whose pullback is $\theta$ since $\Gamma$ is a subgroup of the center. Again, $\theta^{*}$ is fat if $\theta$ is fat and the Weinstein invariants are the same up to a constant.

Finally, if $G$ is a product group, $G=G_{1} \times G_{2}$, or a local product $G=G_{1} \cdot G_{2}=$ $\left(G_{1} \times G_{2}\right) / \Gamma$, then from (1.4) we get, up to a factor,

$$
q_{\left(y_{1}, y_{2}\right)}\left(\alpha_{1}, \alpha_{2}\right)=\sum_{i=0}^{m}\left(\begin{array}{c}
m \\
i
\end{array}\right) q_{y_{1}}^{i}\left(\alpha_{1}\right) q_{y_{2}}^{m-i}\left(\alpha_{2}\right),
$$

where $q_{y_{j}}^{k}$ are given by (1.4) for each $G_{j}$ (we know they are nonzero only for $k=m$ ). In particular, if we change the biinvariant metric on $G$ by multiplying by a constant $c_{i}$ on each factor $G_{i}$, the Weinstein invariants change by a constant as well, once we replace $\left(\alpha_{1}, \alpha_{2}\right)$ by $\left(c_{1} \alpha_{1}, c_{2} \alpha_{2}\right)$, and its nonvanishing is thus independent of the choice of biinvariant metrics.

We now discuss the case of a fiber bundle $\pi: M \rightarrow B$ with fiber $F$, where we allow a general Riemannian submersion with totally geodesic fibers. The fiber bundle $\pi$ is associated to a $G$-principal bundle $\tau: P \rightarrow B$ via $M=P \times_{G} F$, where $G$ acts on $P$ on the right and on $F$ on the left, $[(p, h)]=\left\{\left(p g, g^{-1} h\right): g \in G\right\}$, and $\pi$ can be regarded 
as the projection onto the first factor. Choose a principal connection $\theta: T P \rightarrow \mathfrak{g}$, a metric on $F$ invariant under the action of $G$ and a metric on $B$. The horizontal space at $p \in P$ given by $\mathcal{H}_{p}=\operatorname{ker} \theta_{p}$ defines a horizontal space at $x=[(p, h)] \in M$ via $\mathcal{H}_{[(p, h)]}=\left[\left(\mathcal{H}_{p}, 0\right)\right]$. We now define a metric on $M$ by pulling back the metric on $B$ with $\pi$, by declaring the fibers to be orthogonal to $\mathcal{H}$, and choosing the metric on $\pi^{-1}(b) \simeq F: p \rightarrow[(p, h)], p \in \tau^{-1}(b)$, to be the given metric on $F$. In this metric, $\pi$ is a Riemannian submersion with totally geodesic fibers isometric to $F$, and any Riemannian submersion with totally geodesic fibers arises in this fashion for some principal bundle; see Ziller [21] and Gromoll and Walschap [9] for details. Notice also that, in contrast to Weinstein [19], this metric does not require a choice of metrics on $G$ or $P$. The metrics described as above are often called connection metrics of the fiber bundle.

Bérard-Bergery showed in [2] that the holonomy group of a fat fiber bundle acts isometrically and transitively on the fibers. Since the holonomy group is contained in $G, G$ acts transitively on the fibers as well. Hence we can assume that $F=G / H$ for some subgroup $H$ and $M=P \times_{G} G / H=P / H$. If $\mathfrak{h}^{\perp} \subset \mathfrak{g}$ is the orthogonal complement of the Lie algebra $\mathfrak{h}$ of $H$ with respect to our fixed auxiliary biinvariant metric on $G$, Weinstein showed:

The connection metric on $\pi: M=P \times_{G} G / H \rightarrow B$ is fat if and only if $\Omega$ is $\mathfrak{h}^{\perp}$-fat.

That is, $\Omega_{u}$ is nondegenerate on $\mathcal{H}$ for all $0 \neq u \in \mathfrak{h}^{\perp}$. Notice that this is again independent of the $G$-invariant metric on $F=G / H$ and the metric on $B$, in other words, it only depends of the principal connection. Therefore, fatness of $\pi$ implies the nonvanishing of the Weinstein invariants of the $G$-principal bundle associated to any $0 \neq y \in \mathfrak{h}^{\perp}$, and we write these in terms of the characteristic numbers of the $G$-principal bundle.

If the left action of $G$ on $G / H$ extends to an action of $G^{\prime}$ with $G / H=G^{\prime} / H^{\prime}$, one can view, as above, the metric on $M$ induced by $\theta$ as the metric induced by the unique extension of the principal connection $\theta$ to a connection $\theta^{\prime}$ on $P^{\prime}=P \times_{G} G^{\prime}$. Furthermore, it follows that if $\theta$ is fat then $\theta^{\prime}$ is fat as well. Indeed, on $P \subset P^{\prime}$, $\theta=\left.\theta^{\prime}\right|_{P}$ and thus $\Omega=\left.\Omega^{\prime}\right|_{P}$, in particular, $\left.\Omega^{\prime}\right|_{P}$ has values in $\mathfrak{g} \subset \mathfrak{g}^{\prime}$ and using the identification $T F=\mathfrak{g}^{\perp} \simeq \mathfrak{g}^{\perp}$ it follows that $\Omega$ is $\mathfrak{h}^{\perp}$-fat if and only if $\left.\Omega^{\prime}\right|_{P}$ is $\mathfrak{h}^{\perp}$-fat. Thus the possible principal bundles are all extensions of the holonomy bundle and the principal connection is the uniquely defined extension of the tautological principal connection of the holonomy bundle. Nevertheless, the Weinstein invariants depend on the particular choice of the principal bundle, a fact that we will be able to exploit in certain situations. 


\subsection{Symmetric polynomials and Schur functions}

Fix a positive integer $n \in \mathbb{N}$. A base of the algebra of symmetric polynomials in $x=\left(x_{1}, \ldots, x_{n}\right)$ is indexed by partitions $\lambda=\left(\lambda_{1}, \ldots, \lambda_{n}\right) \in \mathbb{N}_{0}^{n}=(\mathbb{N} \cup\{0\})^{n}$, where $\lambda$ is nonincreasing, ie, $\lambda_{i} \geq \lambda_{i+1}$. Denote by

$$
K_{m}=\left\{\lambda \in \mathbb{N}_{0}^{n}: \lambda_{1} \geq \cdots \geq \lambda_{n},|\lambda|=m\right\}
$$

the set of partitions of degree $m$, where for each $\mu \in \mathbb{N}_{0}^{n}$ we set $|\mu|=\sum_{j=1}^{n} \mu_{j}$. We also set $\mu !=\mu_{1} ! \cdots \mu_{n}$ ! and $x^{\mu}=x_{1}^{\mu_{1}} \cdots x_{n}^{\mu_{n}}$ for $x \in \mathbb{R}^{n}$. We use the convention $K_{m}=\varnothing$ if $m$ is not an integer. For $k \in \mathbb{N}_{0}$ we also denote by $k=(k, \ldots, k) \in \mathbb{N}_{0}^{n}$ and $k \mu=\left(k \mu_{1}, \ldots, k \mu_{n}\right)$. The notation $\mu \subset \gamma$ means that $\mu_{i} \leq \gamma_{i}$ for all $i$. We say that $\mu$ is even (resp. odd) if each $\mu_{i}$ is even (resp. odd). The partition $\lambda^{\prime}$ conjugate to $\lambda \in K_{m}$ is the partition $\lambda^{\prime} \in \mathbb{N}_{0}^{m}$ defined as $\lambda_{i}^{\prime}=\#\left\{j: \lambda_{j} \geq i\right\}, 1 \leq i \leq m$. Since $\lambda^{\prime} \subset n$, the set of conjugate partitions to $K_{m}$ is

$$
K_{m}^{\prime}=\left\{\lambda \in \mathbb{N}_{0}^{m}: n \geq \lambda_{1} \geq \cdots \geq \lambda_{m},|\lambda|=m\right\} .
$$

A basic property of the conjugated partitions is that $\lambda^{\prime \prime}=\lambda$ for $\lambda \in K_{m}$, so $K_{m}^{\prime \prime}=K_{m}$. For $\lambda \in K_{m}^{\prime}$, we denote by $n-\lambda$ the partition $\left(n-\lambda_{m}, n-\lambda_{m-1}, \ldots, n-\lambda_{1}\right)$.

Associated with each $\mu \in \mathbb{N}_{0}^{n}$ there is an alternant $A_{\mu}$ defined by

$$
A_{\mu}(x)=\operatorname{det}\left(x_{i}^{\mu_{j}}\right)=\sum_{\sigma \in S_{n}} \operatorname{sign}(\sigma) x^{\sigma \mu} .
$$

By definition one has $A_{\sigma \mu}=\operatorname{sign}(\sigma) A_{\mu}$ for all $\sigma$ in the permutation group $S_{n}$ of $n$ elements. Since $A_{\mu}=0$ when $\mu$ has repeated indexes, a nonvanishing $A_{\mu}$ can be written, up to sign, as $A_{\lambda+\rho}$ for $\lambda \in K_{m}$, where $m=|\mu|-n(n-1) / 2$ and

$$
\rho=\rho_{n}:=(n-1, n-2, \ldots, 1,0) .
$$

As a special case we have the Vandermonde determinant

$$
\Delta(x):=A_{\rho}(x)=\operatorname{det}\left(x_{i}^{j-1}\right)=\prod_{i<j}\left(x_{i}-x_{j}\right) .
$$

Since, for any partition $\lambda, A_{\lambda+\rho}(x)=0$ if $x_{i}=x_{j}$ for some $i \neq j$, we have that $\Delta$ divides $A_{\lambda+\rho}$. This allows us to define the degree $m$ homogeneous symmetric Schur polynomial

$$
S_{\lambda}:=A_{\lambda+\rho} / \Delta, \quad \lambda \in K_{m}
$$

The complete homogeneous symmetric polynomial of degree $m$ in $n$ variables is defined as

$$
h_{m}=h_{m}(x):=\sum_{|\mu|=m} x^{\mu},
$$


and we set $h_{\lambda}=\prod_{i} h_{\lambda_{i}}$ for any partition $\lambda$. The elementary symmetric polynomials are

$$
\sigma_{m}:=\sum_{|\mu|=m, \mu \subset 1} x^{\mu}
$$

There is a simple relation between these polynomials given by

$$
h_{0}=1, \quad \sum_{j=0}^{r}(-1)^{j} \sigma_{j} h_{r-j}=0 \quad \forall r \geq 1,
$$

where we set $\sigma_{m}=0$ for $m>n$ or $m<0$ (cf Macdonald [12, $\left(2.6^{\prime}\right)$ ) or Fulton and Harris [8]). It follows that $\left\{h_{0}, h_{1}, \ldots, h_{n}\right\}$ is another basis of the algebra of symmetric polynomials. Moreover, one has

$$
h_{m}=\operatorname{det}\left(\sigma_{j-i+1}\right)_{1 \leq i, j \leq m} \quad \forall m \in \mathbb{N}
$$

(cf [12, page 20]). For the special cases $n=2$ or $x_{3}=\cdots=x_{n}=0$ we also have that

$$
h_{m}=\frac{1}{2^{m}} \sum_{j=0}^{[m / 2]}\left(\begin{array}{c}
m+1 \\
2 j+1
\end{array}\right) \sigma_{1}^{m-2 j}\left(\sigma_{1}^{2}-4 \sigma_{2}\right)^{j} .
$$

Indeed, by definition, $h_{m}=\left(x_{1}^{m+1}-x_{2}^{m+1}\right) /\left(x_{1}-x_{2}\right)$. If we consider $x_{1}$ and $x_{2}$ formally as the roots of $x^{2}-\sigma_{1} x+\sigma_{2}=0$ and apply the binomial theorem one obtains (1.9).

Remark 1.10 Because of (1.8), when $E$ is a complex vector bundle, $h_{m}$ represents the $m$-Chern class $c_{m}\left(-E^{*}\right)$ of the formal negative of the dual of $E$.

We summarize now the main properties of Schur functions that will be needed.

Proposition 1.11 We have the following properties of Schur functions $S_{\lambda}\left(x_{1}, \ldots, x_{n}\right)$ for $\lambda \in K_{m}$ :

(a) $S_{\lambda}=\operatorname{det}\left(h_{\lambda_{i}+j-i}\right)_{1 \leq i, j \leq n}$.

(b) $S_{\lambda}=\operatorname{det}\left(\sigma_{\lambda_{i}^{\prime}+j-i}\right)_{1 \leq i, j \leq m}$, where $\lambda^{\prime} \in K_{m}^{\prime}$ is the conjugate partition of $\lambda$.

(c) $S_{\lambda}=\sigma_{m}$ for $\lambda=(1, \ldots, 1,0, \ldots, 0)$, where there are $m 1$ 's.

(d) $S_{\lambda}=h_{m}=\operatorname{det}\left(\sigma_{j-i+1}\right)_{1 \leq i, j \leq m}$ for $\lambda=(m, 0, \ldots, 0)$.

(e) $S_{\lambda}=\sigma_{n}^{\lambda_{n}} S_{\tilde{\lambda}}$, where $\tilde{\lambda}=\lambda-\lambda_{n} 1$.

(f) $S_{0}=1$.

(g) $S_{\lambda}\left(e_{1}\right)=1$ for $\lambda=(m, 0, \ldots, 0)$ and 0 for any other partition $\lambda$.

(h) $S_{\lambda}\left(e_{1}+t e_{2}\right)=\sum_{i=k}^{m-k} t^{i}$, if $\lambda=(m-k, k, 0, \ldots, 0), 0 \leq k \leq[m / 2]$, and 0 for any other partition $\lambda$. 
Proof All statements are immediate consequences of the well known Jacobi-Trudi identities (a) and (b) (see [12, (3.4) and (3.5)]).

We will see in the next section that Weinstein invariants can be explicitly written in terms of Schur functions and then, by part (b) of Proposition 1.11, in terms of Chern and Pontrjagin numbers.

\section{Weinstein invariants for the classical groups}

Assume $G$ is connected. To convert the integral in (1.4) into a sum, we use a formula due to Harish-Chandra (see Warner [18, Theorem 3.2.1.3]):

$$
t^{r} \pi(y) \pi(x) \int_{G} e^{t\left\langle\operatorname{Ad}_{g}(y), x\right\rangle}=\sum_{w \in W} \operatorname{det}(w) e^{t\langle w x, y\rangle}, \quad t \in \mathbb{R},
$$

which holds after multiplying the biinvariant metric with a suitable constant. Here, $W$ denotes the Weyl group of $G, \pi$ the product of the positive roots of $(\mathfrak{g}, \mathfrak{t}), r$ the number of positive roots, ie, $2 r=\operatorname{dim} G-\operatorname{rank}(G)$, and $x, y \in \mathfrak{t} \cong \mathbb{R}^{n}$. Comparing Taylor series expansions in $t$, we have from (1.4) that

$$
\begin{aligned}
\pi(x) \pi(y) q_{\mathfrak{o}}^{k}(x) & =\frac{k !}{(k+r) !} \sum_{w \in W} \operatorname{det}(w)\langle w x, y\rangle^{k+r} \\
& =k ! \sum_{w \in W} \operatorname{det}(w) \sum_{|\mu|=k+r} \frac{1}{\mu !}(w x)^{\mu} y^{\mu} \\
& =\sum_{|\mu|=k+r} \frac{k !}{\mu !} y^{\mu} \sum_{w \in W} \operatorname{det}(w)(w x)^{\mu}
\end{aligned}
$$

with $k \in \mathbb{N}, \mu \in \mathbb{N}_{0}^{n}$. Therefore,

where

$$
\pi(x) \pi(y) q_{\mathfrak{o}}^{k}(x)=\sum_{|\mu|=k+r} \frac{k !}{\mu !} y^{\mu} L_{\mu}(x),
$$

$$
L_{\mu}(x):=\sum_{w \in W} \operatorname{det}(w)(w x)^{\mu}
$$

We now compute the polynomials $q_{y}^{k}, y \in \mathfrak{t}$, for the classical groups in terms of Schur functions. Since they are $\operatorname{Ad}_{G}$-invariant we only need to describe their restriction to $t$. Recall that $K_{r}$ is empty if $r$ is not an integer, and we set a sum over the empty set to be zero. 
Proposition 2.2 Let $G$ be one of the classical groups with Lie algebra $\mathfrak{g}$ and $\mathfrak{t} \cong \mathbb{R}^{n}$ the Lie algebra of a maximal torus of $G$. Then, up to a positive constant which only depends on $G$, we have the following expressions for $q_{y}^{k}: \mathfrak{t} \rightarrow \mathbb{R}$ for each $y \in \mathfrak{t}$ and $k \in \mathbb{N}$ :

(a) If $G=\mathrm{U}(n)$ or $\mathrm{SU}(n)$, then

$$
q_{y}^{k}(x)=\sum_{\lambda \in K_{k}} \frac{k !}{(\lambda+\rho) !} S_{\lambda}(y) S_{\lambda}(x) .
$$

(b) If $G=\mathrm{O}(2 n), \mathrm{O}(2 n+1), \mathrm{SO}(2 n+1)$ or $\mathbb{S}(n)$, then

$$
q_{y}^{k}(x)=\sum_{\lambda \in K_{k / 2}} \frac{k !}{(2(\lambda+\rho)+\epsilon) !} S_{\lambda}\left(y^{2}\right) S_{\lambda}\left(x^{2}\right),
$$

where $\epsilon=0$ for $G=\mathrm{O}(2 n)$ and $\epsilon=1$ otherwise.

(c) If $G=\mathrm{SO}(2 n)$, then

$$
\begin{aligned}
q_{y}^{k}(x)= & \sum_{\lambda \in K_{k / 2}} \frac{k !}{(2(\lambda+\rho)) !} S_{\lambda}\left(y^{2}\right) S_{\lambda}\left(x^{2}\right) \\
& +\sum_{\lambda \in K_{(k-n) / 2}} \frac{k !}{(2(\lambda+\rho)+1) !} \sigma_{n}(y) S_{\lambda}\left(y^{2}\right) \sigma_{n}(x) S_{\lambda}\left(x^{2}\right) .
\end{aligned}
$$

Proof We proceed case by case since the actual expressions in (2.1) involve the structure of the Lie algebra of $G$.

$G=\mathrm{U}(n)$. The Weyl group $W=S_{n}$ acts on $\mathfrak{t}$ as the permutation group $S_{n}$ of $n$ elements. Furthermore, $r=n(n-1) / 2, \pi(x)=\Delta(x)$ and $L_{\mu}(x)=A_{\mu}(x)$. Since $L_{\mu}=0$ if there are two repeated integers in $\mu$ we obtain from (2.1) that

$$
\pi(x) \pi(y) q_{y}^{k}(x)=\sum_{\mu-\rho \in K_{k}} \frac{k !}{\mu !} \sum_{\tau \in S_{n}} y^{\tau \mu} L_{\tau \mu}(x)=\sum_{\mu-\rho \in K_{k}} \frac{k !}{\mu !} A_{\mu}(y) A_{\mu}(x),
$$

which proves Proposition 2.2 (a) for the unitary group.

$G=\mathrm{SU}(n)$. Identifying the maximal torus of $\mathrm{SU}(n)$ with $\left\{x \in \mathbb{R}^{n}: \sigma_{1}(x)=0\right\}$, the same formula as for $\mathrm{U}(n)$ holds simply taking into account that $\sigma_{1}(x)=\sigma_{1}(y)=0$, since the Weyl group and the roots of $\mathrm{SU}(n)$ coincide with those of $\mathrm{U}(n)$.

$G=\operatorname{SO}(2 n)$. Here, $W=S_{n} \times \mathbb{Z}_{2}^{n-1}$ acts on $\mathfrak{t}$ as the permutation group and by an even change of signs, and $r=n(n-1)$. Observe that $L_{\mu}=0$ if $\mu$ contains both an even and an odd index or when two indexes are repeated, and $L_{\mu}=2^{n-1} A_{\mu}$ otherwise. 
On the other hand we have $L_{2 \mu}(x)=L_{\mu}\left(x^{2}\right)=2^{n-1} A_{\mu}\left(x^{2}\right)$ and $L_{2 \mu+1}(x)=$ $\sigma_{n}(x) L_{\mu}\left(x^{2}\right)=2^{n-1} \sigma_{n}(x) A_{\mu}\left(x^{2}\right)$. Therefore,

$$
\begin{aligned}
\pi(x) \pi(y) q_{y}^{k}(x)= & \sum_{|\mu|=k+r, \mu \text { even or odd }} \frac{k !}{\mu !} \sum_{\tau \in S_{n}} y^{\tau \mu} L_{\tau \mu}(x) \\
= & \sum_{\mu-\rho \in K_{k / 2}} \frac{k ! 2^{n-1}}{(2 \mu) !} A_{\mu}\left(y^{2}\right) A_{\mu}\left(x^{2}\right) \\
& +\sigma_{n}(x) \sigma_{n}(y) \sum_{\mu-\rho \in K_{(k-n) / 2}} \frac{k ! 2^{n-1}}{(2 \mu+1) !} A_{\mu}\left(y^{2}\right) A_{\mu}\left(x^{2}\right) .
\end{aligned}
$$

We immediately get Proposition 2.2 (c) up to a factor $2^{n-1}$ since for $\operatorname{SO}(2 n)$ we have $\pi(x)=\Delta\left(x^{2}\right)$.

$G=\mathrm{SO}(2 n+1)$. Here, $W=S_{n} \times \mathbb{Z}_{2}^{n}$ acts on $\mathfrak{t}$ as the permutation group and by arbitrary sign changes, and $r=n^{2}$. Thus, $L_{\mu}=0$ if $\mu$ contains an even index, and $L_{2 \mu+1}(x)=\sigma_{n}(x) L_{\mu}\left(x^{2}\right)=2^{n} \sigma_{n}(x) A_{\mu}\left(x^{2}\right)$. Since $\pi(x)=\sigma_{n}(x) \Delta\left(x^{2}\right)$, we get Proposition 2.2 (b) for $\mathrm{SO}(2 n+1)$ up to a factor $2^{n}$.

$G=\mathrm{O}(2 n+\epsilon), \epsilon=0,1$. These groups share a maximal torus with $\mathrm{SO}(2 n+\epsilon)$. However, we cannot apply Harish-Chandra's formula directly, since the orthogonal group is not connected. Write $G=G_{0} \cup g^{\prime} G_{0}$ where $G_{0}=\operatorname{SO}(2 n+\epsilon)$ and $g^{\prime}=$ $\operatorname{diag}(-1,1, \ldots, 1) \in G \backslash G_{0}$. From (1.4) we get

$$
q_{y}^{k}(x)=\int_{G}\left\langle\operatorname{Ad}_{g}(y), x\right\rangle^{k} d g=\hat{q}_{y}^{k}(x)+\hat{q}_{y}^{k}\left(\operatorname{Ad}_{g^{\prime}}(x)\right),
$$

where $\hat{q}_{y}^{k}(x)=\int_{G_{0}}\left\langle\operatorname{Ad}_{g}(y), x\right\rangle^{k} d g$. Since $\operatorname{Ad}_{g^{\prime}}$ preserves $S_{\lambda}\left(x^{2}\right)$ and changes the sign of $\sigma_{n}(x)$, Proposition 2.2 (b) and (c) for $\mathrm{SO}(2 n+\epsilon)$ imply Proposition 2.2 (b) for the orthogonal group up to a factor $2^{n+\epsilon}$.

$G=\mathbb{S}(n)$. The symplectic group $\mathbb{S}(n)$ shares with $\mathrm{U}(n)$ the same maximal torus. The Weyl group acts on it in the same way as the one of $\operatorname{SO}(2 n+1)$, but $\pi(x)=$ $2^{n} \sigma_{n}(x) \Delta\left(x^{2}\right)$. Therefore, the expression differs from the one for $\operatorname{SO}(2 n+1)$ only by a $2^{n}$ factor.

We now have all the ingredients needed to express Weinstein invariants in terms of Chern and Pontrjagin numbers. Recall that for any fat fiber bundle where the fibers are different from $\mathbb{S}^{1}$ the dimension of the base must be divisible by 4 .

Theorem 2.3 Let $G$ be one of the classical groups and $\mathfrak{t} \cong \mathbb{R}^{n}$ a maximal abelian subalgebra of its Lie algebra. Let $G \rightarrow P \rightarrow B^{2 m}$ be a $G$-principal bundle and an 
element $y \in \mathfrak{t}$ that is fat. Taking into account that in all statements the indexes $i, j$ of the matrices run over $1 \leq i, j \leq r$ for $\lambda \in K_{r}^{\prime}$, we have:

(a) If $G=T^{n}$, then

$$
\left(\sum_{i=1}^{n} y_{i} c_{i}\right)^{m} \neq 0
$$

where $c_{1}, \ldots, c_{n} \in H^{2}(B, \mathbb{Z})$ are the Chern classes of $P$.

(b) If $G=\mathrm{U}(n)$ or $\mathrm{SU}(n)$, then

$$
\sum_{\lambda \in K_{m}^{\prime}}\left(n-\lambda+\rho_{m}\right) ! \operatorname{det}\left(\sigma_{\lambda_{i}+j-i}(y)\right) \operatorname{det}\left(c_{\lambda_{i}+j-i}\right) \neq 0,
$$

where $c_{k} \in H^{2 k}(B, \mathbb{Z})$ is the $k-$ th Chern class of $P$, with $\sigma_{1}(y)=0$ and $c_{1}=0$ for $G=\mathrm{SU}(n)$.

(c) If $G=\mathrm{O}(2 n), \mathrm{O}(2 n+1), \mathrm{SO}(2 n+1)$ or $\mathbb{S}(n)$, then

$$
\sum_{\lambda \in K_{m / 2}^{\prime}}\left(2\left(n-\lambda+\rho_{m / 2}\right)+\epsilon\right) ! \operatorname{det}\left(\sigma_{\lambda_{i}+j-i}\left(y^{2}\right)\right) \operatorname{det}\left(p_{\lambda_{i}+j-i}\right) \neq 0
$$

where $p_{k} \in H^{4 k}(B, \mathbb{Z})$ is the $k$-th Pontrjagin class of $P$, with $\epsilon=0$ for $G=\mathrm{O}(2 n)$ and $\epsilon=1$ otherwise.

(d) If $G=\mathrm{SO}(2 n)$, then

$$
\begin{aligned}
& \sum_{\lambda \in K_{m / 2}^{\prime}} \frac{\left.2\left(n-\lambda+\rho_{m / 2}\right)\right) !}{\left(2 \rho_{(m+2 n) / 2}\right) !} \operatorname{det}\left(\sigma_{\lambda_{i}+j-i}\left(y^{2}\right)\right) \operatorname{det}\left(p_{\lambda_{i}+j-i}\right) \\
+ & e \sum_{\lambda \in K_{(m-n) / 2}^{\prime}} \frac{\left(2\left(n-\lambda+\rho_{(m-n) / 2}\right)+1\right)}{\left(2 \rho_{(m+n) / 2}+1\right)} \sigma_{n}(y) \operatorname{det}\left(\sigma_{\lambda_{i}+j-i}\left(y^{2}\right)\right) \operatorname{det}\left(p_{\lambda_{i}+j-i}\right) \neq 0,
\end{aligned}
$$

where $e \in H^{2 n}(B, \mathbb{Z})$ is the Euler class of $P$.

Proof For the torus, the Weyl group $W$ is trivial and we simply get, using Fubini and (1.4), that

$$
q_{\mathfrak{o}}(x)=\left(\sum_{i=1}^{n} y_{i} x_{i}\right)^{m}
$$

which gives us part (a).

Using that $\lambda^{\prime \prime}=\lambda \in K_{m}$ and Proposition 1.11 (b), the other cases are direct consequences of Proposition 2.2 for $k=m$, writing the expressions in terms of conjugate 
partitions, and using that $\rho_{m+n} !=(\lambda+\rho) !\left(n-\lambda^{\prime}+\rho_{m}\right) !$ and hence $\left(2 \rho_{m+n}+\epsilon\right) !=$ $\left.(2(\lambda+\rho)+\epsilon) !\left(2\left(n-\lambda^{\prime}+\rho_{m}\right)+\epsilon\right)\right)$ !. Indeed, this follows from the fact that

$$
\left\{\lambda_{i}+n-i: 1 \leq i \leq n\right\} \cup\left\{n+j-\lambda_{j}^{\prime}-1: 1 \leq j \leq m\right\}=\{0,1,2, \ldots, m+n-1\},
$$

with the union being disjoint; see $[12,(1.7)]$.

Remark 2.4 Weinstein invariants for $G=G_{2}$. Our methods apply to all Lie groups, and not only to the classical ones. For example, regard $G=G_{2}$ as a subgroup of $\mathrm{SO}(7)$, with its maximal torus being the subset of the maximal torus of $\mathrm{SO}(7)$ such that $x_{1}+x_{2}+x_{3}=0$. It is convenient to write $3 s_{i}=2 x_{i}-x_{j}-x_{k}$ with $\{i, j, k\}=\{1,2,3\}$ and notice that $s_{1}+s_{2}+s_{3}=0$. The positive roots are given by $s_{i}, 1 \leq i \leq 3$ and $s_{j}-s_{k}, j<k$, and so $\pi(s)=\sigma_{3}(s) \Delta(s)$. The Weyl group $W=S_{3} \times \mathbb{Z}_{2}$ acts by permutations and simultaneous sign change on the $s_{i}$ 's. Therefore, $L_{\mu}(s)=\left(1+(-1)^{|\mu|}\right) \sum_{\sigma \in S_{3}} \operatorname{sign}(\sigma)(\sigma s)^{\mu}$ and $L_{\tau \mu}=\operatorname{sign}(\tau) L_{\mu}$ for all $\tau \in S_{3}$. So, taking into account that $\sigma_{1}(y)=\sigma_{1}(s)=0$,

$$
\begin{aligned}
q_{\mathfrak{o}}(s) & =(\pi(y) \pi(s))^{-1} \sum_{\lambda \in K_{m+3}} \frac{2 m !}{(\lambda+\rho) !} A_{\lambda+\rho}(y) A_{\lambda+\rho}(s) \\
& =\sum_{\lambda \in K_{m+3}} \frac{2 m !}{(\lambda+\rho) !} \frac{S_{\lambda}(y)}{\sigma_{3}(y)} \frac{S_{\lambda}(s)}{\sigma_{3}(s)} .
\end{aligned}
$$

These invariants can be written in terms of $\sigma_{2}(s) \in H^{4}(B, \mathbb{Z})$ and $\sigma_{3}\left(s^{2}\right) \in H^{12}(B, \mathbb{Z})$ which form a base of the $\operatorname{Ad}_{G_{2}}$-invariant polynomials. Indeed, since $\sigma_{1}(s)=0$, $\sigma_{1}\left(s^{2}\right)=-2 \sigma_{2}(s)$ and $\sigma_{2}(s)^{2}=\sigma_{2}\left(s^{2}\right)$; see eg [11].

\section{First applications}

In this section we prove Theorem 1 and Theorem 2, and provide several applications of the explicit expressions of the Weinstein invariants to the case of low rank groups. In the process, we generalize and prove some of the corollaries stated in the Introduction.

\section{$3.1 G=T^{n}$}

In this subsection, we provide the proof of Theorem 1 in the Introduction by means of a well-known algebraic result in the theory of isometric rigidity of submanifolds.

Let $\beta: V \times V^{\prime} \rightarrow W$ be a bilinear map between real vector spaces. For $y \in V^{\prime}$, define $\beta_{y}: V \rightarrow W$ as $\beta_{y}(x)=\beta(x, y)$. The set $\operatorname{RE}(\beta)=\left\{y \in V^{\prime}: \operatorname{rank} \beta_{y} \geq \operatorname{rank} \beta_{z}, \forall z \in V^{\prime}\right\}$ is clearly open and dense in $V^{\prime}$. The following result is essentially contained in Moore [14]. 
Lemma 3.1 One has $\beta_{z}\left(\operatorname{ker} \beta_{y}\right) \subset \operatorname{Im} \beta_{y}$ for all $y \in \operatorname{RE}(\beta), z \in V^{\prime}$.

Proof If $t$ is small, $t z+y \in \operatorname{RE}(\beta)$. Then, $I_{t}=\operatorname{Im} \beta_{t z+y}$ converges to $I_{0}=\operatorname{Im} \beta_{y}$ as $t \rightarrow 0$. But if $x \in \operatorname{ker} \beta_{y}, \beta_{z}(x)=\beta_{t z+y}\left(t^{-1} x\right) \in I_{t}$ for all $t$ small, $t \neq 0$. Hence, $\beta_{z}(x) \in \operatorname{Im} \beta_{y}$.

Proof of Theorem 1 Fix $n, s \in \mathbb{N}$, and let $V \subseteq H^{s}(B, \mathbb{R})$ be a subspace satisfying $c^{k} \neq 0$ for all $c \in V \backslash\{0\}$. Consider the map $\alpha: V \times H^{s(r-1)}(B, \mathbb{R}) \rightarrow H^{s r}(B, \mathbb{R})$ given by $\alpha(c, b)=c b$, where the product is the cup product of the de Rham cohomology ring of $B$. We have:

Claim If $b \in \operatorname{RE}(\alpha)$ and $r<k$, the map $\alpha_{b}: V \rightarrow H^{s r}(B, \mathbb{R})$ is a monomorphism.

To prove the Claim, take $w \in \operatorname{ker} \alpha_{b} \subseteq V$. By Lemma 3.1 we have that $w^{r}=$ $\alpha_{w^{r-1}}(w) \in \operatorname{Im} \alpha_{b}$. Hence, there is $v \in V$ such that $w^{r}=v b$. But then $w^{r+1}=$ $v b w= \pm v \alpha_{b}(w)=0$. Since $w \in V$, we get $w=0$ and the Claim is proved.

Theorem 1 is now a consequence of the above Claim applied to the linear subspace $V^{n} \subset H^{2}(B, \mathbb{R})$ spanned by the Chern classes of the bundle, ie by the pull back of $H^{2}\left(B_{T^{n}}\right)$ under the classifying map.

\section{$3.2 G=\mathbf{U}(n)$}

The general expression of the Weinstein invariants involves several determinants and hence are difficult to use. But for certain vectors $y \in \mathfrak{g}$ it can be simplified. It is thus useful to express the nonvanishing of the invariants for some particular cases in a more explicit way.

Proposition 3.2 Let $P$ be a $y$-fat $\mathrm{U}(n)$-principal bundle over a compact manifold $B^{2 m}$ for $y \in \mathfrak{t} \subset \mathfrak{u}(n)$. Denoting $h_{k}=\operatorname{det}\left(c_{j-i+1}\right)_{1 \leq i, j \leq k}$ we have the following:

(a) If $y=(1, \ldots, 1)$, then $c_{1}^{m} \neq 0$.

(b) If $y=(1,0, \ldots, 0)$, then $h_{m} \neq 0$.

(c) If $y=(1+t, 1, \ldots, 1)$, then

$$
\sum_{k=0}^{m}\left(\begin{array}{l}
m+n-1 \\
n+k-1
\end{array}\right) t^{k} c_{1}^{m-k} h_{k} \neq 0 .
$$


(d) If $y=(1, t, 0 \ldots, 0)$, then

$$
\sum_{k=0}^{m / 2}\left(\begin{array}{c}
m+2 n-3 \\
n+k-2
\end{array}\right)\left(\sum_{i=k}^{m-k} t^{i}\right)\left(h_{k} h_{m-k}-h_{k-1} h_{m-k+1}\right) \neq 0 .
$$

In particular, restrictions (c) for $t=-n$ and (d) for $t=-1$ also apply for $\mathrm{SU}(n)-$ principal bundles.

Proof Part (a) follows directly from the definition of the Weinstein invariant (1.4) since $q_{\mathfrak{o}}(\alpha)=\int_{G}\langle y, \alpha\rangle^{m} d g=\int_{G} \operatorname{tr}(\alpha)^{m} d g=c_{1}^{m}$.

For part (b) apply Proposition 2.2 (a) to $\mathfrak{o}=\operatorname{Ad}_{\mathrm{U}(n)}\left(e_{1}\right)$. Using Proposition $1.11(\mathrm{~g})$ and (d) we obtain

$$
q_{y}=\frac{(n-1) ! m !}{\rho !(m+n-1) !} h_{m}
$$

and hence $q_{y} \neq 0$ is equivalent to $h_{m} \neq 0$.

To prove (c) we use (1.4), part (a), the proof of (b) and Fubini to obtain

$$
\begin{aligned}
q_{y}(\alpha) & =\int_{G}\left(\left\langle\left(e_{1}+\cdots+e_{n}\right), \alpha\right\rangle+t\left\langle e_{1}, \alpha\right\rangle\right)^{m} d g \\
& =\sum_{k=0}^{m}\left(\begin{array}{l}
m \\
k
\end{array}\right) \frac{(n-1) ! k !}{\rho !(k+n-1) !} t^{k} c_{1}^{m-k} h_{k},
\end{aligned}
$$

and thus $\quad q_{y}(\alpha)=\frac{(n-1) ! k ! m !}{\rho !(m+n-1) !} \sum_{k=0}^{m}\left(\begin{array}{c}m+n-1 \\ n+k-1\end{array}\right) t^{k} c_{1}^{m-k} h_{k}$.

This proves part (c).

Finally, to prove (d), by Proposition 2.2 (a) and Proposition 1.11 (h), we have

$$
q_{y}=\sum_{\lambda} \frac{m !}{(\lambda+\rho) !} S_{\lambda}(y) S_{\lambda}(x)
$$

for $\lambda=(m-k, k, 0, \ldots, 0), k=0, \ldots, m / 2$. Proposition 1.11 (a) and (h) then imply

$$
q_{y}(\alpha)=\frac{(n-1) !(n-2) ! m !}{\rho !(m+2 n-3) !} \sum_{k=0}^{m / 2}\left(\begin{array}{c}
m+2 n-3 \\
n+k-2
\end{array}\right) t^{k} c_{1}^{m-k} h_{k} .
$$

which finishes our proof. 


\section{$3.3 G=\mathrm{U}(2)$}

We now derive a formula for the Weinstein invariants for $G=\mathrm{U}(2)$ which is simpler than the one obtained from Proposition 3.2 in the case of $n=2$. This in particular proves Theorem 2 for $G=\mathrm{U}(2)$.

Proposition 3.3 Let $\mathrm{U}(2) \rightarrow P \rightarrow B^{2 m}$ be a principal bundle. If $y=(1,-1)$ is fat then $\left(c_{1}^{2}-4 c_{2}\right)^{m / 2} \neq 0$, while if $y=(1+t, 1-t)$ is fat for some $t \in \mathbb{R}$ then

$$
\sum_{j=0}^{m / 2}\left(\begin{array}{c}
m+1 \\
2 j+1
\end{array}\right) t^{2 j} c_{1}^{m-2 j}\left(c_{1}^{2}-4 c_{2}\right)^{j} \neq 0 .
$$

Proof We have 2-fold covers $\pi_{1}: S^{1} \times \mathrm{SU}(2) \rightarrow \mathrm{U}(2),(z, A) \rightarrow z A$ and $\pi_{2}: \mathrm{U}(2) \rightarrow$ $S^{1} \times \mathrm{SO}(3)$, obtained by dividing by $\pm \mathrm{Id}$. All 3 have the same polynomials $q_{y}$. The restrictions of $\pi_{2} \circ \pi_{1}$ to $S^{1}$ and $\mathrm{SU}(2)$ are both 2 fold covers. On the maximal torus level we clearly have $\left(\pi_{2} \circ \pi_{1}\right)_{*}(a, b)=(2 a, 2 b)$ and $\left(\pi_{1}\right)_{*}(a, b)=(a+b, a-b)$. Thus $\left(\pi_{2}\right)_{*}(s, t)=(s+t, s-t)$. Now for the Chern class polynomials of $\mathrm{U}(2)$ we have $c_{1}=s+t$ and $c_{2}=s t$ and thus $c_{1}^{2}-4 c_{2}=(s-t)^{2}$. Hence $c_{1}$ becomes the Euler class for the $S^{1}$ factor in $S^{1} \times \mathrm{SO}(3)$ and $c_{1}^{2}-4 c_{2}$ the Pontrjagin class $p_{1}$ for $\mathrm{SO}(3)$. For the Weinstein polynomials of $S^{1}$ we have $q_{y}^{k}=y^{k} c_{1}^{k}$ and for $\mathrm{SO}(3)$, using Proposition $2.2(\mathrm{~b}), q_{y}^{k}=(2 /(k+1)) y^{k} p_{1}^{k / 2}$ if $k$ is even and 0 otherwise. Since $\left(\pi_{2}\right)_{*}(1+t, 1-t)=(2,2 t),(1.4)$ and Fubini imply

$$
\begin{aligned}
q_{y}(\alpha) & =\sum_{j=0}^{m / 2}\left(\begin{array}{l}
m \\
2 j
\end{array}\right) c_{1}^{m-2 j} \frac{2^{m}}{2 j+1} t^{2 j}\left(c_{1}^{2}-4 c_{2}\right)^{j} \\
& =\frac{2^{m}}{m+1} \sum_{j=0}^{m / 2}\left(\begin{array}{c}
m+1 \\
2 j+1
\end{array}\right) t^{2 j} c_{1}^{m-2 j}\left(c_{1}^{2}-4 c_{2}\right)^{j} .
\end{aligned}
$$

The case of $y=(1,-1)$ follows by considering $y / t=(1 / t+1,1 / t-1)$ and letting $t \rightarrow \infty$ in (3.4).

As we observed in Section 1 , if $P \rightarrow B$ is a $(1+t, 1-t)-$ fat $\mathrm{U}(2)$ bundle, then $P /\{ \pm \mathrm{Id}\} \rightarrow B$ is a $(1, t)$-fat $S^{1} \times \mathrm{SO}(3)$ bundle and the claim follows.

Remark 3.5 The proof shows that $c_{1}^{2}-4 c_{2}$ is the first Pontrjagin class of the $\mathrm{SO}(3)=\mathrm{U}(2) / Z(\mathrm{U}(2))$ bundle $P / Z(\mathrm{U}(2))$, and $c_{1}$ the Euler class of the circle bundle $P / \mathrm{SU}(2)$.

As a consequence, we obtain the following result. The assumption is eg satisfied when $b_{4}\left(B^{2 m}\right)=1$. This also proves Corollary 1 for $\mathrm{U}(2)$. 
Corollary 3.6 Let $\mathrm{U}(2) \rightarrow P \rightarrow B^{2 m}$ be a principal bundle for which $c_{1}^{2}=r\left(c_{1}^{2}-4 c_{2}\right)$ for some $r \in \mathbb{R}$. We then have:

(a) If $\left(c_{1}^{2}-4 c_{2}\right)^{m / 2}=0$, all Weinstein invariants vanish.

(b) If $\left(c_{1}^{2}-4 c_{2}\right)^{m / 2} \neq 0$ and $r=0$, there is exactly one adjoint orbit whose Weinstein invariant vanishes.

(c) If $\left(c_{1}^{2}-4 c_{2}\right)^{m / 2} \neq 0$ and $r<0$, there are exactly $m / 2$ adjoint orbits whose Weinstein invariants vanish.

(d) If $\left(c_{1}^{2}-4 c_{2}\right)^{m / 2} \neq 0$ and $r>0$, no Weinstein invariant vanishes.

In particular, if the bundle is fat, then $\left(c_{1}^{2}-4 c_{2}\right)^{m / 2} \neq 0$ and $r>0$.

Proof We use Proposition 3.3. For $r=0$, the vector $y=(1,1)$, ie $t=0$, is clearly not fat. If $t \neq 0,(3.4)$ is equivalent to $\left(c_{1}^{2}-4 c_{2}\right)^{m / 2} \neq 0$. For $r \neq 0$ we obtain $\left(c_{1}^{2}-4 c_{2}\right)^{m / 2} \neq 0$ when $t=0$. When $t \neq 0$ we note that

$$
(1+z)^{k+1}-(1-z)^{k+1}=2 z \sum_{j=0}^{[k / 2]}\left(\begin{array}{c}
k+1 \\
2 j+1
\end{array}\right) z^{2 j} \quad \forall z \in \mathbb{C}, k \in \mathbb{N} .
$$

Hence the vector $y=(1+t, 1-t), t \neq 0$, is fat if and only if

$$
(\sqrt{r}+t)^{m+1} \neq(\sqrt{r}-t)^{m+1} .
$$

If $r>0$ this is satisfied for all $t \in \mathbb{R}$. If, on the contrary, $r<0$, then one easily sees that there are exactly $m / 2$ positive values of $t$ that satisfy the equality in (3.7), and thus $y=(1+t, 1-t)$ cannot be fat. Notice also that $y$ and $(1-t, 1+t)$ lie in the same adjoint orbit.

We point out that it is easy to state and prove similar results to both Proposition 3.3 and Corollary 3.6 for $m$ odd.

For the proof of Corollary 6 , let $\mathfrak{s}$ be the Lie algebra of $S_{p, q}^{1}$, where we can assume $p \geq 1$ and $q \leq p$ by reversing the roles of $p$ and $q$ or the orientation of the circle. Since $\mathfrak{s}=\mathbb{R}(p, q)$, a straightforward computation shows that

$$
\operatorname{Ad}_{\mathrm{U}(2)}\left(\mathfrak{s}^{\perp}\right)=\mathbb{R} \bigcup_{t \geq|p+q|} \operatorname{Ad}_{\mathrm{U}(2)}(p-q+t, p-q-t) .
$$


Proof of Corollary 6 If $q=p=1$, we simply get from (3.4) that $\left(c_{1}^{2}-4 c_{2}\right)^{m / 2} \neq 0$. If $q \neq p$, by (3.8) we need (3.4) for $t \geq|(p+q) /(p-q)|$. This is equivalent to (3.7) for $t \geq|(p+q) /(p-q)|$, which is in turn easily seen to be equivalent to

$$
r>-\left(\frac{1-\cos \left(\frac{\pi}{m+1}\right)}{1+\cos \left(\frac{\pi}{m+1}\right)}\right)\left(\frac{p+q}{p-q}\right)^{2} .
$$

Remark 3.9 In particular, if $m=2, \mathfrak{s}^{\perp}$-fatness implies that $c_{1}^{2}=r\left(c_{1}^{2}-4 c_{2}\right)$ with $3 r>-((p+q) /(p-q))^{2}$. In [21] the results in [5] were applied to such lens space bundles as well. It was shown that, for some orientation of the bundle, $P_{+}:=P / \mathrm{SU}(2)$ is fat and for $P_{-}:=P / Z\left(\mathrm{U}(2)\right.$ we have $\left|p_{1}\left(P_{-}\right)\right|<((p+q) /(p-q))^{2} p_{1}\left(P_{+}\right)$. Since $p_{1}\left(P_{+}\right)=c_{1}^{2}$ and $p_{1}\left(P_{-}\right)=c_{1}^{2}-4 c_{2}$ or vice versa, Corollary 6 for $m=2$ and the result in [21] complement each other. In particular, if $p+q=0$, no fat principal connection exists, while for any other pair $p, q$ there exist fat lens space bundles over $\mathbb{C} P^{2}$; see Section 6.

The analysis of the Weinstein invariants is easy when the dimension of the base is small:

Fat U(2)-bundles over 8-dimensional manifolds For $m=4$ and $y=(1+t, 1-t)$ we get from Proposition 3.3 (a) that $\left(c_{1}^{2}-4 c_{2}\right)^{2} t^{4}+10 c_{1}^{2}\left(c_{1}^{2}-4 c_{2}\right) t^{2}+5 c_{1}^{4} \neq 0$ for all $t \in \mathbb{R}$, while for $y=(1,-1)$ we have $\left(c_{1}^{2}-4 c_{2}\right)^{2} \neq 0$. Therefore, no Weinstein invariant vanishes if and only if

$$
5\left(c_{1}^{2}\left(c_{1}^{2}-4 c_{2}\right)\right)^{2}<c_{1}^{4}\left(c_{1}^{2}-4 c_{2}\right)^{2}
$$

or $c_{1}^{4},\left(c_{1}^{2}-4 c_{2}\right)^{2}$ and $c_{1}^{2}\left(c_{1}^{2}-4 c_{2}\right)$ don't vanish and have the same sign.

\section{$3.4 G=\operatorname{SO}(4)$}

As for the $\mathrm{U}(2)$ case, for $\mathrm{SO}(4)$ we can provide a simpler expression for the invariants. In particular, this proves Theorem 2 for $\mathrm{SO}(4)$.

Proposition 3.10 Let $\mathrm{SO}(4) \rightarrow P \rightarrow B^{2 m}$ be a principal bundle. If $(1,-1)$ is fat then $\left(p_{1}+2 e\right)^{m / 2} \neq 0$, while if $(1+t, 1-t)$ is fat for some $t \in \mathbb{R}$, we have

$$
\sum_{j=0}^{m / 2}\left(\begin{array}{c}
m+2 \\
2 j+1
\end{array}\right) t^{2 j}\left(p_{1}-2 e\right)^{m / 2-j}\left(p_{1}+2 e\right)^{j} \neq 0 .
$$


Proof As in the proof of Proposition 3.3 we have 2 -fold covers $\pi_{1}: \mathbb{S}(1) \times \mathbb{S}(1) \rightarrow$ $\mathrm{SO}(4),\left(q_{1}, q_{2}\right) \rightarrow\left\{v \rightarrow q_{1} v q_{2}^{-1}\right\}$ using multiplication of quaternions and $\pi_{2}: \mathrm{SO}(4) \rightarrow$ $\mathrm{SO}(3) \times \mathrm{SO}(3)$, obtained by dividing by $-\mathrm{Id}$. The restrictions of $\pi_{2} \circ \pi_{1}$ to each $\mathbb{S}(1)$ factor are again 2 fold covers and hence $\left(\pi_{2} \circ \pi_{1}\right)_{*}(a, b)=(2 a, 2 b)$. We also have $\left(\pi_{1}\right)_{*}(a, b)=(a+b, a-b)$ and thus $\left(\pi_{2}\right)_{*}(s, t)=(s+t, s-t)$. Since $p_{1}=s^{2}+t^{2}$ and $e=s t$ it follows that $p_{1} \pm 2 e=(s \pm t)^{2}$ are the Pontrjagin classes of the two $\mathrm{SO}(3)$ factors. We thus have

$$
\left.q_{y}(\alpha)=2^{m} \sum_{k=0}^{m / 2}\left(\begin{array}{l}
m \\
2 k
\end{array}\right) \frac{2}{m-2 k+1}\left(p_{1}-2 e\right)^{m-2 k} \frac{2}{2 k+1} t^{2 k}\left(p_{1}+2 e\right)\right)^{k}
$$

which, up to a factor $2^{m+2} /((m+1)(m+2))$ is the expression in Proposition 3.10 .

Remark 3.11 The proof shows that $p_{1} \pm 2 e$ is the first Pontrjagin class of the $\mathrm{SO}(3)=$ $\mathrm{SO}(4) / \mathrm{SU}(2)_{ \pm}$principal bundles $P / \mathrm{SU}(2)_{\mp}$, where $\mathrm{SU}(2)_{-}$and $\mathrm{SU}(2)_{+}$are the two normal subgroups of $\mathrm{SO}(4)$.

With the same argument as in the proof of Corollary 3.6 we easily prove the following, which in particular generalizes Corollary 1 for $\mathrm{SO}(4)$.

Corollary 3.12 Let $\mathrm{SO}(4) \rightarrow P \rightarrow B^{2 m}$ be a principal bundle for which $p_{1}+2 e=$ $r\left(p_{1}-2 e\right)$ for some $r \in \mathbb{R}$. Then one of the following holds:

(a) If $\left(p_{1}-2 e\right)^{m / 2}=0$, then all Weinstein invariants vanish.

(b) If $\left(p_{1}-2 e\right)^{m / 2} \neq 0$ and $r=0$, then there is exactly one adjoint orbit whose Weinstein invariant vanishes.

(c) If $\left(p_{1}-2 e\right)^{m / 2} \neq 0$ and $r<0$, then there are exactly $m / 2$ adjoint orbits whose Weinstein invariant vanishes.

(d) If $\left(p_{1}-2 e\right)^{m / 2} \neq 0$ and $r>0$, then no Weinstein invariant vanishes.

In particular, if the bundle is fat, then $\left(p_{1}-2 e\right)^{m / 2} \neq 0$ and $r>0$.

Remark 3.13 In contrast to the U(2) case, it is easy to see that $\mathfrak{s}^{\perp}$-fatness implies full $\mathfrak{s o}(4)$-fatness for the Lie algebra $\mathfrak{s}$ of $S_{p, q}^{1} \subset \mathrm{SO}(4)$. In fact, even $\mathfrak{t}^{\perp}$-fatness implies $\mathfrak{s o}(4)$-fatness, since $\mathfrak{t} \subset \operatorname{Ad}_{\mathrm{SO}(4)}\left(\mathfrak{t}^{\perp}\right)$. Actually, the latter property seems to hold for all semisimple Lie groups of rank $>1$.

From the dimension restriction it follows that for a fat $\mathrm{SO}(4)$ bundle $\operatorname{dim} B$ must be divisible by 8 . In the lowest dimensional case we have: 
Fat $\mathrm{SO}(4)$-bundles over 8-dimensional manifolds When $n=2$ and $m=4$, Proposition 3.10 for $y=(1+t, 1-t)$ gives

$$
3\left(p_{1}-2 e\right)^{2}+10 t^{2}\left(p_{1}-2 e\right)\left(p_{1}+2 e\right)+3 t^{4}\left(p_{1}+2 e\right)^{2} \neq 0 \quad \forall t \in \mathbb{R},
$$

while for $y=(1,-1)$ we have $\left(p_{1}+2 e\right)^{2} \neq 0$. Thus, no Weinstein invariant vanishes if and only if

$$
25\left(p_{1}^{2}-4 e^{2}\right)^{2}<9\left(p_{1}+2 e\right)^{2}\left(p_{1}-2 e\right)^{2}
$$

or

$\left(p_{1}-2 e\right)^{2},\left(p_{1}+2 e\right)^{2}$ and $\left(p_{1}+2 e\right)\left(p_{1}-2 e\right)$ don't vanish and have the same sign.

\section{$3.5 G=\mathrm{SU}(3)$}

We analyze one further case of rank 2 groups, those with $G=\mathrm{SU}(3)$, in order to illustrate the difficulties one faces for other Lie groups if one wants to express the restrictions for full fatness purely in terms of characteristic numbers. By (1.2), the lowest dimensional case is already $\operatorname{dim} B=32$. Here, the invariants for $\mathrm{SU}(3)$ reduce to

$$
\begin{aligned}
& c_{2}^{2}\left(\left(511 t^{12}+3066 t^{11}+8814 t^{10}+15965 t^{9}+21798 t^{8}+25128 t^{7}+26583 t^{6}\right.\right. \\
& \left.+25128 t^{5}+21798 t^{4}+15965 t^{3}+8814 t^{2}+3066 t+511\right) c_{2}^{6} \\
& +\left(1917 t^{12}+11502 t^{11}-15876 t^{10}-184815 t^{9}-498150 t^{8}-757188 t^{7}-834867 t^{6}\right. \\
& \left.\quad-757188 t^{5}-498150 t^{4}-184815 t^{3}-15876 t^{2}+11502 t+1917\right) c_{3}^{2} c_{2}^{3} \\
& \left.+729\left(t^{4}+2 t^{3}-6 t^{2}-7 t+1\right)\left(t^{4}+11 t^{3}+21 t^{2}+11 t+1\right)\left(t^{4}-7 t^{3}-6 t^{2}+2 t+1\right) c_{3}^{4}\right) \neq 0,
\end{aligned}
$$

for all $0 \leq t \leq 1$. Here we can restrict ourselves to $t \leq 1$ since if $t$ is a root, then $1 / t$ also is a root. In particular, $c_{2}^{2}\left(15 c_{3}^{4}-21 c_{3}^{2} c_{2}^{3}+c_{2}^{6}\right)$ and $c_{2}^{2}\left(729 c_{3}^{4}+1917 c_{3}^{2} c_{2}^{3}+511 c_{2}^{6}\right)$ do not vanish and have the same sign.

Now, if $c_{3}^{2}=r c_{2}^{3}$, we write the above as $a(t) r^{2}+b(t) r+c(t) \neq 0$. It is easy to see that the function $r_{-}(t)=\left(-b-\sqrt{b^{2}-4 a c}\right) / 2 a$ has only one essential singularity in $[0,1]$ at $t_{0} \cong 0.12920428615$, for which $\lim _{t \rightarrow t_{0}^{+}} r_{-}(t)=+\infty$ and $\lim _{t \rightarrow t_{0}^{-}} r_{-}(t)=-\infty$. Therefore, the function $r_{-}(t)$ for $t \in\left[0, t_{0}\right) \cup\left(t_{0}, 1\right]$ takes values in $\left(-\infty, r_{2}\right] \cup$ $\left[r_{1},+\infty\right)$, where $r_{2}=r_{-}(0)=(-71-9 \sqrt{37}) / 54 \cong-2.3286$ and $r_{1}:=r_{-}(1)=$ $(15309-\sqrt{202479021}) / 21870$. The same argument for $r_{+}(t)=\left(-b+\sqrt{b^{2}-4 a c}\right) / 2 a$ allows us to conclude:

Full fatness and $c_{3}^{2}=r c_{2}^{3}$ implies that $-0.30102106 \cong r_{0}<r<r_{1} \cong 0.0493593$, where $r_{0}:=r_{+}(0)=(-71+9 \sqrt{37}) / 54$. 
A particular interesting case are $\mathrm{SU}(3) / T^{2}$ fiber bundles since this is one of the positively curved Wallach flag manifolds. But $\mathfrak{t}^{\perp}$-fatness coincides with full $\mathfrak{s u}(3)$ fatness since it is easy to check that $\operatorname{Ad}_{\mathrm{SU}(3)}\left(\mathfrak{t}^{\perp}\right)=\mathfrak{s u}(3)$. Hence we also have that for any circle $S_{p, q}^{1} \subset \mathrm{SU}(3)$ that $\mathfrak{s}^{\perp}$-fatness implies full fatness.

\section{Fat sphere bundles}

In this section we compute the Weinstein invariants for sphere bundles with positive vertizontal sectional curvatures, and provide applications related to partial fatness. We will exclude fat $S^{1}$-fiber bundles which are simply in one-to-one correspondence with symplectic manifolds.

\subsection{Real sphere bundles}

Regard an arbitrary sphere bundle with totally geodesic fibers of dimension $k \geq 2$ as the associated bundle to a principal bundle $\mathrm{O}(k+1) \rightarrow P \rightarrow B^{2 m}$,

$$
\mathbb{S}^{k} \rightarrow P^{\prime}=P \times_{\mathrm{O}(k+1)} \mathrm{O}(k+1) / \mathrm{O}(k) \rightarrow B^{2 m} .
$$

Recall that $P^{\prime}$ has positive vertizontal curvatures if and only if $P$ is $\mathfrak{s o}(k)^{\perp}$-fat. In this situation, since $\operatorname{Ad}_{\mathrm{O}(k+1)}\left(\mathfrak{s o}(k)^{\perp}\right)=\mathbb{R} \operatorname{Ad}_{\mathrm{O}(k+1)}\left(e_{1}\right)$, Proposition 1.11 together with Proposition 2.2 (b) and (c) yield

$$
q_{\mathfrak{s o}(k) \perp}(x)=h_{m / 2}\left(x^{2}\right)=\operatorname{det}\left(p_{j-i+1}\right)_{1 \leq i, j \leq m / 2} \neq 0 .
$$

This proves Corollary 2. In particular, the Weinstein invariant is independent of the dimension of the fibers and for $m \leq 8$ reduces to:

$$
\begin{array}{cl}
\operatorname{dim}(B) & h_{m / 2} \neq 0 \\
\hline 4 & p_{1} \neq 0 \\
8 & p_{2} \neq p_{1}^{2} \\
12 & p_{3} \neq 2 p_{1} p_{2}-p_{1}^{3} \\
16 & p_{4} \neq p_{1}^{4}-3 p_{1}^{2} p_{2}+2 p_{1} p_{3}+p_{2}^{2}
\end{array}
$$

We point out that for orientable bundles, ie, $G=\mathrm{SO}(k+1)$, the same formulas hold, since the term containing the Euler class vanishes.

\subsection{Complex sphere bundles}

A sphere bundle of dimension $2 n-1 \geq 3$, whose underlying vector bundle has a complex structure, can be viewed as associated to a principal bundle $\mathrm{U}(n) \rightarrow P \rightarrow B^{2 m}$,

$$
\mathbb{S}^{2 n-1} \rightarrow P^{\prime}=P \times_{\mathrm{U}(n)} \mathrm{U}(n) / \mathrm{U}(n-1) \rightarrow B^{2 m} .
$$


Then $P^{\prime}$ has positive vertizontal curvatures if and only if $P$ is $\mathfrak{u}(n-1)^{\perp}$-fat. Since $\operatorname{Ad}_{\mathrm{U}(n)}\left(\mathfrak{u}(n-1)^{\perp}\right)=\mathbb{R} \bigcup_{t \leq 0} \operatorname{Ad}_{\mathrm{U}(n)}\left(e_{1}+t e_{2}\right)$, Proposition $3.2(\mathrm{~d})$ gives

$$
\sum_{k=0}^{[m / 2]}\left(\begin{array}{c}
m+2 n-3 \\
n+k-2
\end{array}\right)\left(\sum_{i=k}^{m-k} t^{i}\right)\left(h_{k} h_{m-k}-h_{k-1} h_{m-k+1}\right) \neq 0 \quad \forall t \leq 0,
$$

where $h_{k}=\operatorname{det}\left(c_{j-i+1}\right)_{1 \leq i, j \leq k}$. In particular, for $t=0$ we obtain that

$$
\operatorname{det}\left(c_{j-i+1}\right)_{1 \leq i, j \leq m} \neq 0 .
$$

For complex $\mathbb{S}^{3}$ fiber bundles over a 4-dimensional manifold, ie $n=m=2$, fatness implies that $c_{1}^{2}\left(1+t+t^{2}\right)-c_{2}(1-t)^{2} \neq 0$ for all $t \leq 0$, which one easily sees is equivalent to $c_{1}^{2}=s c_{2}$ with $s<1$ or $s>4$. We can combine this information with the results obtained in [5] for general 3-sphere bundles over a 4 dimensional base. It was shown there that there exists an orientation of the bundle such that one of the $\mathrm{SO}(3)$ principal bundles among $P_{ \pm}:=P / \mathrm{SU}(2)_{\mp}$, say $P_{+}$, is fat and $\left|p_{1}\left(P_{-}\right)\right|<p_{1}\left(P_{+}\right)$. If the sphere bundle is a complex sphere bundle, one has, for some choice of orientation, $p_{1}\left(P_{+}\right)=c_{1}^{2}$ and $p_{1}\left(P_{-}\right)=c_{1}^{2}-4 c_{2}$. The above obstruction implies that $c_{1}^{2}=r\left(c_{1}^{2}-4 c_{2}\right)$ with $3 r>-1$ which thus complements [5].

Proof of Corollary 4 In $[4 ; 21]$ it was shown that the only 3 -sphere bundles over $\mathbb{C} P^{2}$ that can possibly admit a fat connection metric are the complex vector bundles with characteristic classes $\left(c_{1}^{2}, c_{2}\right)=(1,1)$ or $(9, k)$, with $k=1,2,3,4$. Thus combining both obstructions, it follows that only the sphere bundles with $\left(c_{1}^{2}, c_{2}\right)=(9,1)$ or $(9,2)$ could possibly admit fat connection metrics. The bundle with $\left(c_{1}^{2}, c_{2}\right)=(9,3)$ corresponds to the tangent bundle of $\mathbb{C} P^{2}$.

For $\mathbb{S}^{5}$ fiber bundles over 8 -dimensional manifolds we have

$$
\left(t^{4}+t^{3}+t^{2}+t+1\right) c_{1}^{4}-3\left(t^{4}+1\right) c_{1}^{2} c_{2}+(2 t-1)(t-2)(1+t)^{2} c_{1} c_{3}+\left(t^{2}-t+1\right)^{2} c_{2}^{2} \neq 0,
$$

for all $t \leq 0$, while for $\mathbb{S}^{7}$ fiber bundles the Weinstein invariants are

$$
\begin{aligned}
& \left(t^{4}+t^{3}+t^{2}+t+1\right) c_{1}^{4}+\left(2 t^{4}-3 t^{3}-13 t^{2}-3 t+2\right) c_{1} c_{3}+(t-1)^{4} c_{2}^{2} \\
& \quad-\left(3 t^{2}+4 t+3\right)(t-1)^{2} c_{1}^{2} c_{2}-\left(t^{4}-4 t^{3}-4 t^{2}-4 t+1\right) c_{4} \neq 0 \quad \forall t \leq 0
\end{aligned}
$$

In particular, for $B^{8}=\mathbb{C} P^{4}$, the Chern classes of the tangent bundle are $c_{i}=\left(\begin{array}{l}5 \\ i\end{array}\right) x^{i}$ for a generator $x \in H^{2}(B, \mathbb{Z})$, and thus $\left(14 t^{4}+119 t^{3}+219 t^{2}+119 t+14\right) x^{4} \neq 0$. But this polynomial has two real roots in $[-1,0]$, and hence $T_{1} \mathbb{C} P^{4} \rightarrow \mathbb{C} P^{4}$ admits no fat connection metric. Notice that, since the sphere bundle $T_{1} \mathbb{C} P^{n} \rightarrow \mathbb{C} P^{n}$ with $n \neq 1,2,4$ has no fat connection metric already for dimension reasons, and using 
Corollary 4 , it follows that only the unit tangent bundle over $\mathbb{C} P^{1}$ has a fat connection metric.

\subsection{Quaternionic sphere bundles}

A sphere bundle of dimension $4 n-1 \geq 3$, whose underlying vector bundle has a quaternionic structure, can be seen as an associated bundle to a principal bundle $\mathbb{S}(n) \rightarrow P \rightarrow B^{2 m}$,

$$
\mathbb{S}^{4 n-1} \rightarrow P^{\prime}=P \times_{\mathbb{S}(n)} \mathbb{S}(n) / \mathbb{S}(n-1) \rightarrow B^{2 m} .
$$

Then, $P^{\prime}$ has positive vertizontal curvatures if and only if $P$ is $\mathfrak{s p}(n-1)^{\perp}$-fat. Since $\operatorname{Ad}_{\mathbb{S}(n)}\left(\mathfrak{s p}(n-1)^{\perp}\right)=\mathbb{R} \bigcup_{t \in \mathbb{R}} \operatorname{Ad}_{\mathbb{S}(n)}\left(e_{1}+t e_{2}\right)$ we conclude that

$$
\sum_{k=0}^{m / 4}\left(\begin{array}{c}
m+4 n-6 \\
2 n+2 k-3
\end{array}\right)\left(\sum_{s=k}^{m / 2-k} t^{2 s}\right)\left(h_{m / 2-k} h_{k}-h_{m / 2-k+1} h_{k-1}\right) \neq 0 \quad \forall t \in \mathbb{R},
$$

where $h_{k}=\operatorname{det}\left(p_{j-i+1}\right)_{1 \leq i, j \leq k}$, with the $p_{i}$ 's being the quaternionic Pontrjagin classes. In particular for $t=0$ we get $\operatorname{det}\left(p_{j-i+1}\right)_{1 \leq i, j \leq m / 2} \neq 0$. For $n=1$, we simply obtain $p_{1}^{m / 2} \neq 0$ while, for $n \geq 2$, the principal bundle must be $\mathfrak{s p}(2) \subset \mathfrak{s p}(n)$ fat and hence 32 divides $m$.

The groups $G=\mathbb{S}(n) \times S$ for $S=S^{1}, S=\mathbb{S}(1)$ also act on $\mathbb{S}^{4 n-1}$ by $(A, z) \cdot v=$ $A v z^{-1}$. Then, $\mathbb{S}^{4 n-1}=\mathbb{S}(n) \times S / H$ for $H=\mathbb{S}(n-1) \times \Delta S$. Thus $\operatorname{Ad}_{G}\left(\mathfrak{h}^{\perp}\right)=$ $\mathbb{R} \bigcup_{0 \leq t \leq 1} \operatorname{Ad}_{G}\left(e_{1}-t e_{2}, t-1\right)$, and, using Fubini, we get the Weinstein invariants

$$
\begin{aligned}
\sum_{i=0}^{m / 2} \sum_{k=0}^{[m / 4-i / 2]} & \left(\begin{array}{l}
m \\
2 i
\end{array}\right)\left(\begin{array}{c}
m-2 i+4 n-6 \\
2 n+2 k-3
\end{array}\right) \\
& \left.\times\left((t-1)^{2 i} \sum_{s=k}^{m / 2-i-k} t^{2 s}\right) w^{i}\left(h_{m / 2-i-k} h_{k}-h_{m / 2-i-k+1} h_{k-1}\right)\right) \neq 0
\end{aligned}
$$

for all $0 \leq t \leq 1$, where $w=c_{1}^{2}$ for $S=S^{1}$ and $w=p_{1}$ for $S=\mathbb{S}(1)$.

Proof of Corollary $3 \mathbb{S}^{7}$ bundles over $\mathbb{S}^{8}$ are constructed by gluing two copies of $D^{8} \times \mathbb{S}^{7}$ along the boundary $\mathbb{S}^{7} \times \mathbb{S}^{7}$ via $(u, v) \rightarrow\left(u, u^{k} v u^{l}\right)$, where $u, v$ are unit Cayley numbers, and $k, l \in \mathbb{Z}$. This defines the sphere bundle $\mathbb{S}^{7} \rightarrow M_{k, l} \rightarrow \mathbb{S}^{8}$. In [16] it was shown that the characteristic classes of this sphere bundle are $p_{2}=6(k-l)$, $e=(k+l)$. The restriction for real sphere bundles already implies Corollary 3 for $k=l$. If the bundle is a sphere bundle of a quaternionic vector bundle, we just saw that it cannot be fat for dimension reasons. We will now determine which bundles $M_{k, l}$ carry a complex structure since any quaternionic vector bundle is also a complex one. 
From the usual relationship between Chern and Pontrjagin classes of a complex vector bundle it follows that $p_{2}=2 c_{4}$ and $c_{4}=e$. Thus a necessary condition is that $k=2 l$. Admitting a complex structure is the same as a reduction of the structure group from $\mathrm{SO}(8)$ to $\mathrm{U}(4)$ and since bundles over $\mathbb{S}^{8}$ are classified by their gluing map along the equator, we need to determine the image of $i_{*}: \pi_{7}(\mathrm{U}(4)) \rightarrow \pi_{7}(\mathrm{SO}(8))$. For this we use the long homotopy sequence of the fibration

$$
\mathrm{U}(4) \rightarrow \mathrm{SO}(8) \rightarrow \mathrm{SO}(8) / \mathrm{U}(4)=\mathrm{SO}(8) / \mathrm{SO}(6) \mathrm{SO}(2)=G_{2}\left(\mathbb{R}^{8}\right),
$$

where the last equality is due to one of the low dimensional isometries of simply connected symmetric spaces. Now, $G_{2}\left(\mathbb{R}^{8}\right)$ is the base of another fibration, $S^{1} \rightarrow$ $V_{2}\left(\mathbb{R}^{8}\right) \rightarrow G_{2}\left(\mathbb{R}^{8}\right)$, with total space the Stiefel manifold of 2 -frames, and they thus have the same homotopy groups. The low dimensional homotopy groups of the Stiefel manifolds are well known; see eg Paechter [15]. In particular, $\pi_{7}\left(G_{2}\left(\mathbb{R}^{8}\right)\right)=$ $\mathbb{Z} \oplus \mathbb{Z}_{2}, \pi_{8}\left(G_{2}\left(\mathbb{R}^{8}\right)\right)=\mathbb{Z}_{2} \oplus \mathbb{Z}_{2}$ and for the homotopy groups of the Lie groups (see eg Mimura and Toda [13]) we have $\pi_{7}(\mathrm{U}(4))=\mathbb{Z}$ and $\pi_{7}(\mathrm{SO}(8))=\mathbb{Z} \oplus \mathbb{Z}$ with a basis of the latter given by the gluing map. Thus $i_{*}$ is injective, and by the above its image lies in $\mathbb{Z}=\{(2 l, l), l \in \mathbb{Z}\}$. Since $\pi_{6}(\mathrm{U}(4))=0$, the cokernel of $i_{*}$ is $\mathbb{Z} \oplus \mathbb{Z}_{2}$ and hence $\operatorname{Im}\left(i_{*}\right)=\{(2 l, l), l$ even $\}$. We conclude that the complex vector bundles are precisely the ones with $k=2 l$ for $l$ even. Notice that in this case (4.2) does not give a contradiction to fatness.

Among the complex sphere bundles, the ones that carry a quaternionic structure are the ones for which $l$ is divisible by four. To see this, consider the long exact sequence in homotopy of $\mathbb{S}(2) \rightarrow \operatorname{SU}(4) \rightarrow \mathbb{S}^{5}$. Since $\pi_{7}\left(\mathbb{S}^{5}\right)=\mathbb{Z}_{2}$ and $\pi_{8}\left(\mathbb{S}^{5}\right)=\mathbb{Z}_{24}$ [10], it follows that the map from $\pi_{7}(\mathbb{S}(2))=\mathbb{Z}$ to $\pi_{7}(\operatorname{SU}(4))=\mathbb{Z}$ is multiplication by two. This finishes our proof.

\section{Topological reduction}

In [21] it was conjectured that if a $G$-principal bundle admits a fat connection, then the structure group of the bundle cannot be reduced to any proper subgroup $H \subset G$ (where one does not assume that the reduced bundle admits a fat connection). We make here the stronger conjecture that this already holds when the $G$-principal connection is $y$-fat for some $y \in \mathfrak{h}^{\perp}$. In this section we show that this is in fact true when $H$ is a connected normal subgroup of $G$. This shows that partial fatness, in some cases, can be used to show that the structure groups cannot be reduced.

A $G$-principal bundle $\pi: P \rightarrow B$ is classified via its classifying map $\phi_{G}: B \rightarrow B_{G}$, where $B_{G}=E / G$ is the classifying space of the Lie group $G$. The characteristic 
classes can then be viewed as pull backs of cohomology classes in $H^{*}\left(B_{G}, \mathbb{Z}\right)$. If the structure group of $P$ reduces, ie, if there exists a submanifold $P^{\prime} \subset P$ invariant under a subgroup $H \subset G$, then the $H$-principal bundle $P^{\prime}$ is called a reduction of $P$ and we have $P=P^{\prime} \times{ }_{H} G$. We have another classifying map for $P^{\prime}, \phi_{H}: B \rightarrow B_{H}$, and clearly $\phi_{G}=B_{i} \circ \phi_{H}$, where $B_{i}: B_{H} \rightarrow B_{G}$ is induced by the inclusion map $i: H \rightarrow G$. Thus, if $x \in H^{*}\left(B_{G}, \mathbb{Z}\right)$ is a characteristic class with $B_{i}^{*}(x)=0$, then $\phi_{G}^{*}(x)=0$ as well. In some cases we can use the nonvanishing of certain characteristic numbers for a fat principal bundle to show that a reduction to $H$ cannot exist. A special case is the following result:

Theorem 5.1 Let $G \rightarrow P \rightarrow B$ be a principal bundle, and $H \subset G$ a connected normal subgroup with Lie algebra $\mathfrak{h}$. If the bundle reduces to $H$, then the Weinstein invariant associated to $y$ vanishes for all $y \in \mathfrak{h}^{\perp}$. In particular, there are no fat vectors in $\mathfrak{h}^{\perp}$.

Proof Since $H$ is normal, $\mathfrak{h}$ is an ideal and thus $\mathfrak{h}^{\perp}$ is also an ideal. Therefore, $G=$ $H \cdot H^{\prime}$ for some normal subgroup $H^{\prime} \subset G$. Since $H \times H^{\prime}$ is a finite cover of $H \cdot H^{\prime}$, both have the same rational cohomology and hence the classifying spaces also have the same cohomology. The map induced by the inclusion $B_{H}=B_{H \times\{e\}} \rightarrow B_{H \times H^{\prime}}=B_{H} \times B_{H^{\prime}}$ clearly sends the characteristic classes coming from the cohomology of $B_{H^{\prime}}$ to 0 . Thus the Weinstein invariant associated to any $y \in \mathfrak{h}^{\perp}$ vanishes because of (1.6).

Remark 5.2 If $\operatorname{rank}(H)=\operatorname{rank}(G)$, then the kernel of $B_{i}^{*}: H^{*}\left(B_{G}\right) \rightarrow H^{*}\left(B_{H}\right)$ is trivial (see Borel [3]), and thus the above method cannot be applied. Clearly, the bigger the rank difference, the larger the kernel. On the other hand, the bigger $\operatorname{rank}(G)$ is, the more difficult it is to understand the multivariable polynomials defining the Weinstein invariants.

\section{Examples of homogeneous fat fiber bundles}

Apart from the case $G=\mathrm{SO}(2)$, where fatness is in one-to-one correspondence with symplectic forms on the base $B$, the known examples of fat bundles all arise as homogeneous bundles from inclusions $H \subset G \subset L$,

$$
G / H \rightarrow L / H \stackrel{\pi}{\longrightarrow} L / G=B .
$$

The metrics on $L / H$ and $L / G$ are chosen to be $L$ invariant, ie induced by a left invariant metric on $L$, invariant under right translations by $G$. If we assume that in the metric on the Lie algebra $\mathfrak{l}$ of $L$ the subspaces $\mathfrak{h}^{\perp} \cap \mathfrak{g}$ and $\mathfrak{g}^{\perp} \subset \mathfrak{l}$ (defined with respect to a biinvariant metric) are orthogonal to each other, Bérard-Bergery showed in [1] that 
the projection is a Riemannian submersion with totally geodesic fibers. Furthermore, the submersion is fat if and only if $[X, Y] \neq 0$ for all nonvanishing $X \in \mathfrak{h}^{\perp} \cap \mathfrak{g}$ and $Y \in \mathfrak{g}^{\perp}$. In addition, Bérard-Bergery classified all such homogeneous fat bundles.

The above homogeneous bundle $\pi$ is associated to the $G$ principal bundle

$$
G \rightarrow L \stackrel{\sigma}{\longrightarrow} L / G,
$$

since $L \times{ }_{G} G / H=L / H$. If $G$ and $H$ have a normal connected subgroup $K$ in common, and thus $G=K \cdot G^{\prime}$ and $H=K \cdot H^{\prime}$, we can also choose the $G^{\prime}$-principal bundle

$$
G^{\prime}=G / K \rightarrow L / K \stackrel{\sigma}{\longrightarrow} L / G,
$$

since $L \times{ }_{K \cdot G^{\prime}} G^{\prime} / H^{\prime}=L / K \times_{G^{\prime}}\left(G^{\prime} / H^{\prime}\right)$. The obstructions will be expressed in terms of the characteristic numbers $q_{y}$ for $y \in \mathfrak{h}^{\perp}$ (resp. $y \in \mathfrak{h}^{\perp}$ ) of the $G$ (resp. $G / K)$ principal bundle.

Example 1 (Lens space bundles) Given the inclusion of groups

$$
\mathrm{U}(n-1) S_{p, q}^{1} \subset \mathrm{U}(n-1) \mathrm{U}(2) \subset \mathrm{U}(n+1), \quad n \geq 2,
$$

with $S_{p, q}^{1}=\operatorname{diag}\left(z^{p}, z^{q}\right) \subset \mathrm{U}(2)$, we obtain the fiber bundle over the complex Grassmannian of 2-planes in $\mathbb{C}^{n+1}, G_{2}\left(\mathbb{C}^{n+1}\right)=\mathrm{U}(n+1) / \mathrm{U}(n-1) \mathrm{U}(2)$,

$$
\mathrm{U}(n-1) \mathrm{U}(2) / \mathrm{U}(n-1) S_{p, q}^{1} \rightarrow \mathrm{U}(n+1) / \mathrm{U}(n-1) S_{p, q}^{1} \rightarrow G_{2}\left(\mathbb{C}^{n+1}\right),
$$

with fiber $\mathrm{U}(2) / S_{p, q}^{1}=\mathrm{SU}(2) /\left\{\operatorname{diag}\left(z^{p}, z^{q}\right): z^{p+q}=1\right\}$, which is the lens space $S^{3} / \mathbb{Z}_{p+q}$ when $p+q \neq 0$. By changing the order and replacing $z$ by $\bar{z}$ if necessary, we can assume that $p \geq q$ and $p \geq 0$ with $\operatorname{gcd}(p, q)=1$. Bérard-Bergery showed in [1] that this bundle if fat, when both the total space and the base are equipped with a homogeneous metric, if and only if $p q>0$. We will show now that for $p q \leq 0$ there is no fat principal connection (not necessarily homogeneous), which will provide a proof of Corollary 5.

The above bundle can be considered to be associated to the $\mathrm{U}(2)$ principal bundle

$$
\mathrm{U}(2) \rightarrow \mathrm{U}(n+1) / \mathrm{U}(n-1) \rightarrow G_{2}\left(\mathbb{C}^{n+1}\right),
$$

and for the proof of Corollary 5 we need its first and second Chern classes. The cohomology ring of the base has been computed in [3] and is given by

$$
H^{*}\left(G_{2}\left(\mathbb{C}^{n+1}\right), \mathbb{Z}\right)=\left(\mathbb{Z}\left[\sigma_{1}, \sigma_{2}\right] \otimes Z\left[\bar{\sigma}_{1}, \ldots, \bar{\sigma}_{n-1}\right]\right) / Z\left[\widetilde{\sigma}_{1}, \ldots, \widetilde{\sigma}_{n+1}\right],
$$

where the $\sigma_{i}$ 's are the symmetric polynomials in $t_{1}, t_{2}$, the $\bar{\sigma}_{i}$ 's the symmetric polynomials in $t_{3}, \ldots, t_{n+1}$ and the $\tilde{\sigma}_{i}$ 's the symmetric polynomials in $t_{1}, \ldots, t_{n+1}$. Furthermore, $c_{1}=\bar{\sigma}_{1}\left(t_{1}, t_{2}\right)=t_{1}+t_{2}$ and $c_{2}=\bar{\sigma}_{2}\left(t_{1}, t_{2}\right)=t_{1} t_{2}$ are the Chern classes of 
the canonical 2-plane bundle $\xi_{1}$ over $G_{2}\left(\mathbb{C}^{n+1}\right)$ which sends a point into the 2-plane defining it. Similarly, $\bar{c}_{1}, \ldots, \bar{c}_{n-1}$ are the Chern classes of the canonical $(n-1)$-plane bundle $\xi_{2}$ over $G_{2}\left(\mathbb{C}^{n+1}\right)$ which sends a point into the $(n-1)$-plane orthogonal to it. Thus we can also express the cohomology ring as $\mathbb{Z}\left[c_{1}, c_{2}\right] \otimes Z\left[\bar{c}_{1}, \ldots, \bar{c}_{n-1}\right]$ divided by the relationships

$$
\left(1+c_{1}+c_{2}\right) \cup\left(1+\bar{c}_{1}+, \ldots,+\bar{c}_{n-1}\right)=\prod_{i=1}^{i=n-1}\left(1+t_{i}\right)=1,
$$

which can be regarded as the product formula for the trivial bundle $\xi_{1} \oplus \xi_{2}$. Since $\xi_{1}=\mathrm{U}(n+1) \times_{\mathrm{U}(n-1) \mathrm{U}(2)} \mathbb{C}^{2}=\mathrm{U}(n+1) / \mathrm{U}(n-1) \times_{\mathrm{U}(2)} \mathbb{C}^{2}, c_{1}, c_{2}$ are also the Chern classes of the $\mathrm{U}(2)$ bundle (6.1).

The above relationships imply the recursive formula

$$
\overline{c_{k}}=-c_{1} \bar{c}_{k-1}-c_{2} \bar{c}_{k-2}, \quad k \geq 1,
$$

where we set $\bar{c}_{0}=1$ and $\bar{c}_{-1}=\bar{c}_{n}=\bar{c}_{n+1}=\cdots=0$. Notice that this is the same relationship as (1.8) once we replace $\sigma_{1}$ by $-c_{1}$ and $\sigma_{2}$ by $c_{2}$. We can thus expresses the Chern classes $\bar{c}_{i}$ in terms of $c_{i}$, as in the proof of (1.9), and obtain

$$
\bar{c}_{k}=(-1 / 2)^{k} \sum_{j=0}^{[k / 2]}\left(\begin{array}{c}
m+1 \\
2 j+1
\end{array}\right) c_{1}^{k-2 j}\left(c_{1}^{2}-4 c_{2}\right)^{j}, \quad k \geq 1 .
$$

If the lens space bundle is fat, the relationship $\bar{c}_{m}=0$ for $m=2 n-2$ then contradicts (3.4) for $t=1$. But by (3.8), (3.4) is required for all $t \geq|(p+q) /(p-q)|$, and so we must have $|(p+q) /(p-q)|>1$, or equivalently, $p q>0$, as claimed in Corollary 5 .

In the lowest dimensional case $n=2$, the total space is the Aloff-Wallach space $\mathrm{SU}(3) / S_{p, q}^{1}$ with embedding $S_{p, q}^{1}=\operatorname{diag}\left(z^{p}, z^{q}, \bar{z}^{p+q}\right)$, where the bundle is not only fat, but has positive sectional curvature when $p q>0$. The metric is obtained from the biinvariant metric on $\mathrm{SU}(3)$ by shortening in the direction of $\mathrm{U}(2)=$ $\{\operatorname{diag}(A, \operatorname{det} \bar{A}), A \in \mathrm{U}(2)\}$. There are 3 such metrics corresponding to embeddings of $\mathrm{U}(2)$ in different coordinates and by changing the embedding, and replacing $z$ to $\bar{z}$ if necessary, any Aloff-Wallach space with $p q(p+q) \neq 0$ has a lens space fibration with $p q>0$. From the above, it follows that for the other two fibrations there exists no fat connection metric whatsoever. If $n>2$, though, there is only one such fibration.

It is interesting to observe that, for all $n>2$, the total space admits a metric with positive curvature on an open and dense set if $p q<0$ (see Wilking [20]), and a metric with nonnegative curvature and positive at one point if $p q \geq 0$ (see Tapp [17]). But these are Riemannian submersion metrics with respect to different fibrations, where the intermediate group $G$ in the description above is replaced by $\mathrm{U}(n) \mathrm{U}(1)$. They are 
now fibrations over $\mathbb{C} P^{n}$ with fiber a lens space

$$
\mathrm{U}(n) \mathrm{U}(1) / \mathrm{U}(n-1) S_{p, q}^{1}=\mathrm{U}(n) / \mathrm{U}(n-1) \cdot \mathbb{Z}_{q}=S^{2 n-1} / \mathbb{Z}_{q} .
$$

If $q \neq 0$, there exists a metric with the above properties on the total space, such that the projection onto $\mathbb{C} P^{n}$ is a Riemannian submersion. But the fibers are not totally geodesic. Notice also that already from the dimension restriction (1.2) it follows that these bundles cannot have a fat connection metric if $n>2$.

There exists another fat lens space fibration coming from the inclusions

$$
K \times S_{p, q}^{1} \subset K \cdot \mathbb{S}(1) \times S^{1} \subset Q \times S^{1}
$$

where $B^{4 m}=Q / K \cdot \mathbb{S}(1)$ is a quaternionic symmetric space and $S_{p, q}^{1} \subset \mathbb{S}(1) \times S^{1}$ is embedded with slope $(p, q)$. Recall that a symmetric space is called quaternionic if $\mathbb{S}(1)$ acts via the Hopf action on the tangent space of the foot point. Furthermore, in the irreducible case, each simple Lie group $Q$ gives rise to exactly one such a space. The above inclusions induce the fibration

$$
K \cdot \mathbb{S}(1) \times S^{1} / K \times S_{p, q}^{1} \rightarrow Q \times S^{1} / K \times S_{p, q}^{1} \rightarrow Q \times S^{1} / K \cdot \mathbb{S}(1) \times S^{1}=Q / K \cdot \mathbb{S}(1)=B^{4 m},
$$

with fiber $\mathbb{S}(1) \times S^{1} / \times S_{p, q}^{1}=\mathbb{S}(1) /\left\{\zeta^{p}: \zeta \in S^{1}, \zeta^{q}=1\right\}$, ie, a lens space $S^{3} / Z_{q}$. Notice that we can assume $p \neq 0$ since otherwise the circle acts ineffectively on the total space and the base. Furthermore, if $q=0$, base and total space are a product with the circle in $\mathbb{S}(1) \times S^{1}$ and hence in both cases the bundle clearly has no fat principal connection. Bérard-Bergery showed that this lens space bundle is fat if and only if $p q \neq 0$.

Combining both families of examples, one sees that there exist fat lens space bundles over $\mathbb{C} P^{2}$ for all $S_{p, q}^{1}$ when $p+q \neq 0$. In [21] it was shown that for $p+q=0$ there exists no fat connection metric.

Example 2 (An SO(4) principal bundle) Consider the $\mathrm{SO}(4)$ principal bundle

$$
\mathrm{SO}(4) \rightarrow G_{2} \rightarrow G_{2} / \mathrm{SO}(4)
$$

Bérard-Bergery showed that this bundle is $\mathfrak{s u}(2)_{ \pm}^{\perp}$ fat where $\mathrm{SU}(2)_{ \pm}$are the two normal subgroups of $\mathrm{SO}(4)$ corresponding to the image of $S^{3} \times\{e\}$ and $\{e\} \times S^{3}$ under the two fold cover $S^{3} \times S^{3} \rightarrow \mathrm{SO}(4)$. Thus the associated bundles $G_{2} \times \mathrm{SO}(4) \mathrm{SO}(4) / \mathrm{SU}(2)_{ \pm}=$ $G_{2} / \mathrm{SU}(2)_{ \pm} \rightarrow G_{2} / \mathrm{SO}(4)$ are both fat $\mathrm{SO}(3)=\mathrm{SO}(4) / \mathrm{SU}(2)_{ \pm}$principal bundles. 
We now compute $p_{1}$ and $e$ of the $\mathrm{SO}(4)$ bundle (6.2). For this we use the Borel method which we now recall. We have a commutative diagram

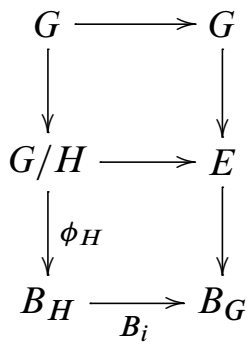

where $i$ is the inclusion $i: H \rightarrow G$. Thus the left hand side $G$ principal bundle is the pull back of the universal bundle on the right. The differentials in the universal spectral sequence are well known and the ones in the left hand side fibration are induced by naturality as soon as we know the map in cohomology $B_{i}^{*}: H^{*}\left(B_{G}\right) \rightarrow H^{*}\left(B_{H}\right)$. The map $\varphi_{H}$ is the classifying map of the $H$ principal bundle $H \rightarrow G \rightarrow G / H$ which can be determined by the edge homomorphism in the spectral sequence and this will then give us the values of the characteristic classes of the $H$ principal bundle.

In order to compute $B_{i}^{*}$, we let $T_{G} \subset G$ and $T_{H} \subset H$ be maximal tori and use the commutativity of the diagram:

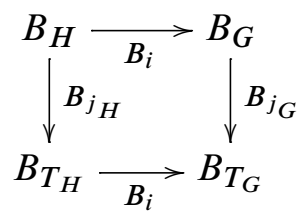

We choose coordinates $\left(t_{1}, \ldots, t_{n}\right)$ of the (integral lattice of the) maximal torus $T_{G} \subset G$ and, by abuse of notation, let $t_{i} \in H^{1}\left(T_{G}, \mathbb{Z}\right)=\operatorname{Hom}\left(\pi_{1}(G), \mathbb{Z}\right)$ and hence $\overline{t_{i}} \in$ $H^{2}\left(B_{T_{G}}\right)$ via transgression in the spectral sequence of the universal bundle of $T_{G}$. We then have $H^{*}\left(B_{T_{G}}\right)=P\left[\bar{t}_{1}, \ldots, \bar{t}_{n}\right]$ and $B_{j_{G}}^{*}$ is injective on the torsion free part of $H^{*}\left(B_{G}\right)$ with image $H^{*}\left(B_{T_{G}}\right)^{W_{G}}$, where $W_{G}$ is the Weyl group of $G$, and similarly for $H$. We thus only need to compute $B_{i}^{*}: H^{*}\left(T_{G}\right) \rightarrow H^{*}\left(T_{H}\right)$, which is easily done.

We now apply all this to $G=G_{2}$ and $H=\mathrm{SO}(4)$. Additional complications arise since the cohomology of the groups and their classifying spaces contain torsion. In [6] it was shown that for both $\mathrm{SO}(4)$ and $G_{2}$, the homomorphism $B_{i_{G}}^{*}: H^{*}\left(B_{G}, \mathbb{Z}\right) \rightarrow$ $H^{*}\left(B_{T_{G}}, \mathbb{Z}\right)$, after dividing by the torsion groups, is injective with image the Weyl group invariant subalgebra. For $G=\mathrm{SO}(4)$, if we use the coordinates for $T_{G}$ as in Section 1 , the transgression $\bar{x}_{i} \in H^{2}\left(B_{T}, \mathbb{Z}\right)=\mathbb{Z} \oplus \mathbb{Z}$ form a basis, and $p_{1}=\bar{x}_{1}^{2}+\bar{x}_{2}^{2}$ and $e=\bar{x}_{1} \bar{x}_{2}$ form a basis of the Weyl group invariant subalgebra. Here $p_{1}$ and $e$ are 
the universal Pontrjagin and Euler classes. There are elements $y_{1}, y_{2} \in H^{3}(G, \mathbb{Z})=$ $\mathbb{Z} \oplus \mathbb{Z}$ such that $d_{3}\left(y_{1}\right)=p_{1}$ and $d_{3}\left(y_{2}\right)=e$ in the universal spectral sequence for $\mathrm{SO}(4)$.

The maximal torus of $G_{2}$ is given by $\left(t_{1}, t_{2}, t_{3}\right)$ with $\sum t_{i}=0$ and a basis of the Weyl group invariant algebra is $x=\frac{1}{2} \sigma_{1}\left(\bar{s}_{i}^{2}\right)$ and $y=\sigma_{3}\left(\bar{s}_{i}^{2}\right)$ where $s_{i}=\frac{1}{3}\left(2 t_{i}-t_{j}-t_{k}\right)$. Since the positive roots are $s_{i}, 1 \leq i \leq 3$ and $s_{j}-s_{k}=t_{j}-t_{k}, j<k$, one easily sees that the roots $s_{3}, s_{1}-s_{2}$ span a subalgebra isomorphic to the Lie algebra of the (unique) $\mathrm{SO}(4)$ in $G_{2}$. In terms of $x_{1}, x_{2}$, the roots are $\pm x_{1} \pm x_{2}$ and hence we can choose $x_{1}=s_{1}, x_{2}=s_{2}$. Thus $p_{1}=s_{1}^{2}+s_{2}^{2}, e=s_{1} s_{2}$ and since $x=\frac{1}{2} \sigma_{1}\left(\bar{s}_{i}^{2}\right)=s_{1}^{2}+s_{2}^{2}+s_{1} s_{2}$ it follows that $d_{4}(x)=p_{1}+e$ in the spectral sequence of the left hand side fibration in the first diagram. Thus $H^{4}\left(G_{2} / \mathrm{SO}(4), \mathbb{Z}\right)=\mathbb{Z}[a]$ with $e=a, p_{1}=-a$ and by Poincaré duality $H^{8}\left(G_{2} / \mathrm{SO}(4), \mathbb{Z}\right)=\mathbb{Z}\left[a^{2}\right]$. Thus $p_{1}+2 e=r\left(p_{1}-2 e\right)$ with $r=-1 / 3$. Hence the $\mathrm{SO}(4)$ principal bundle cannot be fat. Using (3.14), it follows that the Weinstein invariant for $y=(1+t, 1-t)$ is

$$
\left(9-10 t^{2}+t^{4}\right)\left(p_{1}-2 e\right)^{2} \neq 0,
$$

with zeroes $t= \pm 1, \pm 3$ and hence the Weinstein invariant is 0 for the two adjoint orbits with $y=(1,0)$ and $y=(1,-2)$. Notice that the fat bundle by Bérard-Bergery has $y=(1, \pm 1)$. Thus we have:

Corollary 6.3 The principal bundle $\mathrm{SO}(4) \rightarrow G_{2} \rightarrow G_{2} / \mathrm{SO}(4)$ has a homogeneous connection metric which is $(1, \pm 1)$-fat, but admits no $y$-fat principal connection for $y=(1,0)$ or $y=(1,-2)$. In particular, the associated 3-sphere bundle $\mathbb{S}^{3} \rightarrow G_{2} / \mathrm{SO}(3) \rightarrow G_{2} / \mathrm{SO}(4)$ does not admit a fat connection metric.

Example 3 (Sphere bundles) All remaining examples of fat homogeneous fibrations in [1] are bundles with fiber $\mathbb{S}^{n}$ or $\mathbb{R} P^{n}$ represented as $\mathrm{SO}(n) / \mathrm{SO}(n-1)$ or $\mathrm{SO}(n) / \mathrm{O}(n-1)$. We describe next a typical case.

The inclusions $\mathbb{S}(1) \mathbb{S}(1) \mathbb{S}(n-2) \subset \mathbb{S}(2) \mathbb{S}(n-2) \subset \mathbb{S}(n)$ induce the fibration

$$
\mathbb{S}^{4} \rightarrow M \rightarrow G_{2}\left(\mathbb{H}^{n}\right) .
$$

The $\mathbb{S}(2)$ principal bundle $\mathbb{S}(2) \rightarrow \mathbb{S}(n-2) / \mathbb{S}(2) \rightarrow G_{2}\left(\mathbb{H}^{n}\right)$ has quaternionic Pontrjagin classes $p_{1}, p_{2}$ with $H^{*}\left(G_{2}\left(\mathbb{H}^{n}\right), \mathbb{Z}\right)=\mathbb{Z}\left[p_{1}, p_{2}\right] /\left\{\left(1+p_{1}+p_{2}\right) \sum_{i=0}^{i=n-2} \bar{p}_{i}=1\right\}$. If $n=3$ the total space is a positive curved Wallach flag manifold with base $\mathbb{H} P^{2}$. Thus $p_{1}^{2}=p_{2}=1$ in this special case. But as an $\mathbb{S}^{4}$ bundle the structure group is $\mathrm{SO}(5)$ and the 2 -fold cover $\mathbb{S}(2) \rightarrow \mathrm{SO}(5)$ induces a map $H^{*}\left(B_{\mathrm{SO}(5)}\right) \rightarrow H^{*}\left(B_{\mathbb{S}(2)}\right)$ which relates the real and quaternionic Pontrjagin classes. Using this, one easily shows that the real Pontrjagin numbers are $p_{1}^{2}=4$ and $p_{2}=-3$, which is consistent with the obstruction for fat real sphere bundles described above. 


\section{References}

[1] L Bérard-Bergery, Sur certaines fibrations d'espaces homogènes riemanniens, Compositio Math. 30 (1975) 43-61 MR0370432

[2] A L Besse, Einstein manifolds, Ergebnisse der Math. und ihrer Grenzgebiete (3) 10, Springer, Berlin (1987) MR867684

[3] A Borel, Sur la cohomologie des espaces fibrés principaux et des espaces homogènes de groupes de Lie compacts, Ann. of Math. (2) 57 (1953) 115-207 MR0051508

[4] L M Chaves, A theorem of finiteness for fat bundles, Topology 33 (1994) 493-497 MR1286927

[5] A Derdziński, A Rigas, Unflat connections in 3-sphere bundles over $S^{4}$, Trans. Amer. Math. Soc. 265 (1981) 485-493 MR610960

[6] M Feshbach, The image of $H^{*}(B G, \mathbf{Z})$ in $H^{*}(B T, \mathbf{Z})$ for $G$ a compact Lie group with maximal torus $T$, Topology 20 (1981) 93-95 MR592571

[7] L A Florit, W Ziller, Orbifold fibrations of Eschenburg spaces, Geom. Dedicata 127 (2007) 159-175 MR2338524

[8] W Fulton, J Harris, Representation theory: A first course, Graduate Texts in Math. 129, Springer, New York (1991) MR1153249

[9] D Gromoll, G Walschap, Metric foliations and curvature, Progress in Math. 268, Birkhäuser Verlag, Basel (2009) MR2500106

[10] A Hatcher, Algebraic topology, Cambridge Univ. Press (2002) MR1867354

[11] M Kerin, Some new examples with almost positive curvature, Geom. Topol. 15 (2011) 217-260

[12] I G Macdonald, Symmetric functions and Hall polynomials, Oxford Math. Monogr., The Clarendon Press, Oxford Univ. Press, New York (1979) MR553598

[13] M Mimura, H Toda, Topology of Lie groups. I, II, Transl. of Math. Monogr. 91, Amer. Math. Soc. (1991) MR1122592 Translated from the 1978 Japanese edition by the authors

[14] J D Moore, Submanifolds of constant positive curvature. I, Duke Math. J. 44 (1977) 449-484 MR0438256

[15] G F Paechter, The groups $\pi_{r}\left(V_{n, m}\right)$. I, Quart. J. Math. Oxford Ser. (2) 7 (1956) 249-268 MR0131878

[16] N Shimada, Differentiable structures on the 15-sphere and Pontrjagin classes of certain manifolds, Nagoya Math. J. 12 (1957) 59-69 MR0096223

[17] K Tapp, Quasi-positive curvature on homogeneous bundles, J. Differential Geom. 65 (2003) 273-287 MR2058263 
[18] G Warner, Harmonic analysis on semi-simple Lie groups. I, Die Grund. der math. Wissenschaften 188, Springer, New York (1972) MR0498999

[19] A Weinstein, Fat bundles and symplectic manifolds, Adv. in Math. 37 (1980) 239-250 MR591728

[20] B Wilking, Manifolds with positive sectional curvature almost everywhere, Invent. Math. 148 (2002) 117-141 MR1892845

[21] W Ziller, Fatness revisited, Lecture notes, University of Pennsylvania (2001) Available at www.math.upenn.edu/ wziller/papers/Fat-09.pdf

[22] W Ziller, Examples of Riemannian manifolds with non-negative sectional curvature, from: "Surveys in differential geometry. Vol. XI", (J Cheeger, K Grove, editors), Surv. Differ. Geom. 11, Int. Press, Somerville, MA (2007) 63-102 MR2408264

\section{IMPA}

Estrada Dona Castorina, 110, 22460-320 Rio de Janeiro, Brazil

Department of Mathematics, University of Pennsylvania

3451 Walnut Street, Philadelphia PA 19104, USA

luis@impa.br, wziller@math.upenn.edu

http://luis.impa.br

Proposed: Tobias H Colding

Seconded: Peter Teichner, Leonid Polterovich
Received: 19 February 2010 Accepted: 20 April 2011 